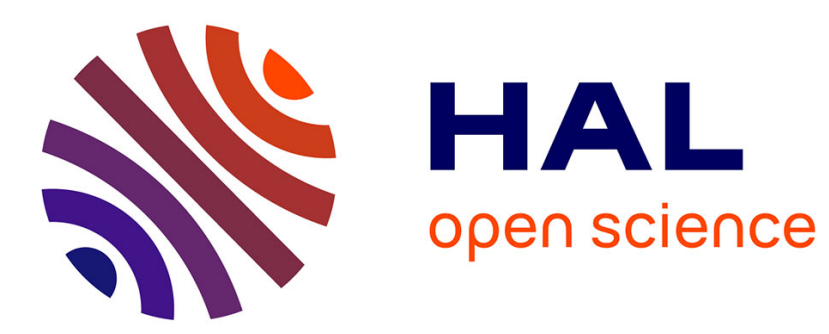

\title{
Numerical Simulation of Wave Impacts with Interfacial Phase Change: An Isothermal Averaged Model
}

\author{
Matthieu Ancellin, Laurent Brosset, Jean-Michel Ghidaglia
}

\section{To cite this version:}

Matthieu Ancellin, Laurent Brosset, Jean-Michel Ghidaglia. Numerical Simulation of Wave Impacts with Interfacial Phase Change: An Isothermal Averaged Model. European Journal of Mechanics B/Fluids, 2018, 72, pp.631-644. 10.1016/j.euromechflu.2018.08.001 . hal-02935980

\section{HAL Id: hal-02935980 \\ https://hal.science/hal-02935980}

Submitted on 10 Sep 2020

HAL is a multi-disciplinary open access archive for the deposit and dissemination of scientific research documents, whether they are published or not. The documents may come from teaching and research institutions in France or abroad, or from public or private research centers.
L'archive ouverte pluridisciplinaire HAL, est destinée au dépôt et à la diffusion de documents scientifiques de niveau recherche, publiés ou non, émanant des établissements d'enseignement et de recherche français ou étrangers, des laboratoires publics ou privés. 


\title{
Numerical Simulation of Wave Impacts with Interfacial Phase Change: An Isothermal Averaged Model
}

\author{
Matthieu Ancellin ${ }^{\mathrm{a}, \mathrm{b}, 1, *}$, Laurent Brosset $^{\mathrm{a}}$, Jean-Michel Ghidaglia ${ }^{\mathrm{b}}$ \\ ${ }^{a}$ GTT (Gaztransport $\&$ Technigaz), Saint-Rémy-lès-Chevreuse, France \\ ${ }^{b}$ CMLA, ENS Paris-Saclay, CNRS, Université Paris-Saclay, Cachan, France
}

\begin{abstract}
Most current numerical simulations on sloshing impact loads do not take into account the possibility of evaporation and condensation of the fluid. Thus, in order to evaluate the influence of phase change the goal of this work is to develop a numerical model able to simulate a wave impact with phase change. Such a model should describe compressible multiphase flows with separate phases, including non-equilibrium interfacial phase change. A first prototype is presented in this paper based on an isothermal Volume-Of-Fluid-type model for the representation of the liquid-vapor interface and a non-conservative advection term for the modelling of phase change. Discretization of the model is made in the Finite Volume framework using a Roe-type scheme. Some original numerical test cases for the validation of the code are discussed and preliminary results for a simplified impact test case are presented.
\end{abstract}

\section{Introduction}

In the transportation of Liquefied Natural Gas (LNG) in tanks on floating structures, such as LNG carriers, one of the principal current technologies is to store the LNG at ambient pressure and cryogenic temperature using a membrane

5 containment system that completely covers the inner sides of the tank walls.

\footnotetext{
* Corresponding author

Email addresses: matthieu.ancellin@ucd.ie (Matthieu Ancellin), lbrosset@gtt.fr (Laurent Brosset), jmg@cmla.ens-cachan.fr (Jean-Michel Ghidaglia)

${ }^{1}$ Present address: School of Mathematics and Statistics, University College Dublin, Ireland
} 
When the sea is rough, the motion of the ship induces significant movement of the liquid in the tank, which can induce violent impacts on the containment system. When designing such containment systems, Gaztransport \& Technigaz (GTT) has to ensure that these impacts do not cause any damage during the working life of the floating structure. Typically, sloshing assessment is based on tests using a model tank, normally at scale 1:40. The tank is filled with water and a heavy gas, and is placed on a hexapod platform which allows it to be moved with six degrees of freedom. The heavy ullage gas is chosen such that the gas-to-liquid density ratio is the same as at full scale. Measurements are made using several pressure sensors that are placed in the impact areas. A complex methodology based on a long-term approach [1] is followed, which enables the probability of failure for any limit state of the containment system to be derived.

However, the complete physics of a real wave impact are not modeled in such an experiment. In particular, phase change (evaporation and condensation) is expected to occur in the full-scale LNG tank since the fluid is close to thermodynamic equilibrium. Unfortunately, its influence on impact loads has not been studied in detail, until now. The final goal of this work is to add a phase change model to a numerical simulation of wave impact and better understand 25 the influence of phase change.

Sloshing tests with boiling water and vapor in conditions close to the phase boundary have been performed [2]. Statistically, the pressure at the impact wall was found to be smaller with water and vapor than with water and noncondensible gases. Hence, the authors attributed the pressure reduction to phase so change.

Simulating a wave impact with phase change adds a new layer of difficulty to an already complex problem. Indeed, the numerical model with phase change should already include complex properties of the two fluids like their compressibility [3]. Furthermore, it is impossible to directly validate such simulations because experimental results of wave impact tests (or sloshing tests with a sin- 
gle impact) show a high variability of the local pressure measurements. This variability is due to the development of free surface instabilities triggered by the escaping gas flow, even when the global wave shape is accurately reproduced (see also for example [4]). More details on wave impact physics can be found in 40 [5].

The only attempts (to our knowledge) to create such a simulation with phase change have been made by [6] for the scenario of a liquid block free falling onto a solid surface and by [7] for two single impact waves obtained by sloshing model tests (without experiment as a reference). Their results seem to be in line with those observed by [2] during sloshing tests with water and vapor.

The problem of phase change influence on sloshing wave impacts is related to the problem of sloshing influence on boil-off evaporation in cryogenic tanks. In the latter boil-off problem, a slow steady heat flow from the exterior of the cryogenic tank can warm up the content of a tank. As a consequence, the liquid evaporates and the pressure rises inside the tank. Sloshing influences this phenomenon, as studied by [6] and [8], among others. However, these problems differ because the spatial scale of the boil-off problem is the size of the tank and the time scale is hours or days, whereas the spatial scale of our problem is the region of impact and the time scale is the duration of the impact. Different modelling approaches should thus be used to describe these two problems.

A great variety of phase change models exists for two-fluid CFD codes, depending on the kind of flow that is being modeled. These models can be sorted using the characteristic size and characteristic duration of the problems they are designed for. The spatial characteristic size of the problem changes the representation of the interface: from a microscopic interface of finite thickness (e.g. [9]) to an industrial averaged macroscopic model (e.g. [10, 11]). The description of interfacial phase change needs to be adapted to the description of the interface. Independently, the characteristic time scale of the problem determines how the relaxation towards liquid-vapor equilibrium is modeled: from instantaneous 
liquid-vapor equilibrium (e.g. [12]) to total non-equilibrium. (Other references used in this work on the modelling of phase change includes [13] and [14].)

The specific space and time scales of the wave impact problem described earlier set the frame of reference of our model. Although a wave impact may involve a complex flow made of droplets and bubbles caused by free surface instabilities, all the numerical studies of wave impacts described previously considered only a relatively smooth zero-thickness interface between pure phases. Existing phase change models at this macroscopic scale include [15] and [16]. However, a wave impact is a very brief and violent phenomenon. Thus, unlike in [15] and [16], both fluids are modeled as compressible and no equilibrium hypothesis is made between phases at the interface.

For slow evaporation and condensation the evolution of the interface can be modelled using a Stefan model [17]. In such a model the mass flow rate can be seen as a Lagrange multiplier ensuring the instantaneous equilibrium condition $p=p^{\text {sat }}(T)$ at the interface. Since an equilibrium hypothesis at the interface so might not be applicable to the wave impact problem, a non-equilibrium model of the Hertz-Knudsen model type [18, 19] will be considered instead. The mass flow rate in this model is proportional to the distance to equilibrium and the interface is brought back to equilibrium in finite time.

In the present paper, the compressible flow with two separate phases is modelled using a Volume-Of-Fluid-type model that is similar to those of [20], [21] or [22]. The liquid gas interface is implicitly captured as liquid-gas mixtures cells, forming a numerically "diffuse" interface, without any special numerical treatment. Although this kind of model can be less accurate than alternatives, such as interface tracking schemes, they are easier to develop and implement, especially in two or three-dimensions. In this paper, our goal is to add nonequilibrium interfacial phase change into such a model. Since diffusion at the interface is a numerical bias, one of the objectives of this work is to ensure that the phase change rate is as independent as possible of the interface profile.

Phase change is a complex process involving pressure variations, large local 

temperature variations are due to the large latent heat released or captured by the mass that changes phase, whereas the pressure variations and velocity jump are due to the density ratio between phases. Correctly including all of this phenomena in a diffuse interface model is a difficult task. During the derivation of our model in the next section, the hypothesis of an isothermal flow will be made to simplify the task. This hypothesis is very strong, as the wave impact cannot be expected to be an isothermal process. However, this work should be seen as only a first step towards more realistic models of wave impacts with phase change. The same kind of approximation for the study of wave impacts is used in other state-of-the-art work, such as [23].

The simplifying assumption of a pure chemical species (either pure water or pure methane) will also be made. Actual LNG is a mixture of several components, where the exact composition has an effect on the phase change behavior of the fluid. However, for the sake of simplicity, multispecies mixtures will not be discussed here.

Only phase change at the interface between liquid and gas are discussed in this paper. Nucleation, that is the appearance of new droplets in the gas or new bubbles in the liquid, is not expected to be necessary for the understanding of the influence of phase change on wave impacts [24,25].

Among the existing compressible-flow models with phase change, the closest to the model presented in this paper are the exact Riemann solver of [26] and [27] and the stiff source term models of [28] and [29]. The former describes the liquid-vapor with phase change by adding one more discontinuity to the solution of the Riemann problem between two phases. This discontinuity follows the change rate. The model presented in this paper gives a similar solution to the Riemann problem (see [24]). However, at the core of our model we place the hyperbolic set of equations instead of the Riemann problem solution. The work of [28] and [29] describe numerically the interface as a liquid-vapor mixture zone 125 in which phase change is modelled as a source term. Our model also involves a 
numerical mixture zone at the interface. Instead of a stiff source term of order zero, we describe phase change using a first order term, as will be seen in the following sections. The behavior of the first order term is expected to be less dependent on numerical diffusion at the interface. The relation between these models and our approach has also been discussed in [30].

In the first few paragraphs of the next section, a model describing the evolution of a flow with two separated phases is presented. Simulation of the model is made using the Finite Volume method, where the model is averaged using a new description of the averaged phase change term. After some simplifications 135 (including the hypothesis of an isothermal flow), a non-conservative hyperbolic system describing the averaged two-phase flow is derived. The numerical scheme used for the simulation of this system is described in Section 3. In particular, the discretization of the non-conservative phase change terms is discussed. The resulting Roe-type scheme is a variant of the scheme proposed in [31].

This scheme is applied in the next section to several simple validation test cases, which illustrate some of the benefits and limitations of the model and its discretization. In particular, it shows that the thickness of the numerical mixture layer at the interface has almost no consequence for the phase change simulation. Finally, in the last section of this paper, the model is applied to idealized wave impact test cases. Preliminary conclusions on the influence of phase change on wave impact loads can then be derived.

The present work is part of a series of contributions to modelling of wave impacts with phase change. The present model has been introduced in [24] along with another interface reconstruction scheme (which will be published in 150 a subsequent article) and a surrogate model updating the model presented in [32]. These different works explore various approaches and shed light on different aspects of phase change modelling. A summary of the models and some partial physical conclusions are presented in [33]. 


\section{Physical model}

155 phase change will be discussed.

\subsection{A sharp interface model}

The two phases are flagged using the order parameter $\chi(x, t)$ that describes the local phase: $\chi=1$ for the gas and $\chi=0$ for the liquid.

At an interface, the evolution of $\chi$ can be written as follows [24]:

$$
\partial_{t} \chi+(u-J / \rho) \cdot \nabla \chi=0,
$$

where the vector $J$ denotes the interfacial surfacic mass flux aligned with the normal vector at the interface. Note that even when both fluids are at rest $(u=0)$, the interface between the two phases can evolve due to phase change.

Using the standard conservative equations for the fluid (mass, momentum and total energy - see (2a) to (2c) below), the balance equations for two fluid phases separated by a free surface can thus be written as:

$$
\begin{aligned}
& \partial_{t} \rho+\nabla \cdot(\rho u)=0, \\
& \partial_{t}(\rho u)+\nabla \cdot(\rho u \otimes u+p \mathbb{I})=0, \\
& \partial_{t}(\rho E)+\nabla \cdot((\rho E+p) u+q)=0, \\
& \partial_{t}(\chi \rho)+\nabla \cdot(\chi \rho u)-J \cdot \nabla \chi=0,
\end{aligned}
$$

where $\rho, u, p$, and $E=e+|u|^{2} / 2$ denote respectively density, velocity, pressure and specific total energy of the fluid. Equation (2d) has been obtained by combining (1) with (2a).

In general, the diffusive heat flux $q$ is given by Fourier's law. More terms can be added on the right-hand side of (2) to describe, for instance, gravity, inertial accelerations or viscosity. In this paper, we focus only on the transport terms of the equations (including the phase change term $J \cdot \nabla \chi$ ).

As discussed in $[24,30]$, this model is hyperbolic without the heat diffusion term. The solution of the Riemann problem for this system contains four waves: 
two sonic waves with velocity $u \pm c$, one contact discontinuity with velocity $u$ and one liquid-vapor interface with velocity $u-J / \rho$. The Rankine-Hugoniot conditions around the latter are the usual boundary conditions around an interface with phase change (see e.g. [34]). If the flow is assumed to be isothermal, this solution is similar to the one described in [26].

We can write

$$
J=J_{l \rightarrow g} \nu_{l \rightarrow g}=J_{l \rightarrow g} \nabla \chi /|\nabla \chi|
$$

where $J_{l \rightarrow g}$ is the evaporation mass flux and $\nu_{l \rightarrow g}$ is the unit normal vector at the interface oriented from the liquid to the gas phase. (Note that the above notation of $|\nabla \chi|_{l \rightarrow g}=\nabla \chi$ as $\nu_{l \rightarrow g}=\nabla \chi /|\nabla \chi|$ should be approached with care since $\nabla \chi$ is a Dirac distribution.) Equation (2d) can then be reformulated as

$$
\partial_{t}(\chi \rho)+\nabla \cdot(\chi \rho u)=J_{l \rightarrow g}|\nabla \chi|
$$

In this form, the phase change term can be understood as a source term for this gas mass balance equation. The term $|\nabla \chi|$ can be seen as an interfacial area term localizing the interface. In this article, the notation of $(2 \mathrm{~d})$ is preferred because it is closer to the form of a usual system of conservation equations.

The extra variable $J$ has been introduced to the equations and thus one more closure relation is needed. This can take the form of an expression relating the interfacial mass flux $J$ to the local thermodynamical state:

$$
J=\mathcal{J}_{l \rightarrow g}\left(p_{g}, T_{g}, p_{l}, T_{l}\right) \nu_{l \rightarrow g},
$$

where $\mathcal{J}_{l \rightarrow g}$ is an expression for the evaporation mass flux as a function of the local pressures and temperatures $p_{g}, T_{g}, p_{l}$ and $T_{l}$, respectively in the gas and the liquid.

The expression for $\mathcal{J}_{l \rightarrow g}$ must be compliant with the second law of thermodynamics to ensure the physical relevance of the model (and thus the good behavior of the numerical code). This point will not be discussed in more depth in this paper. Later in this paper, we will focus on an isothermal evolution at temperature $T_{0}$ and the mass flux across the interface will thus be of the form

$$
J \propto\left(p^{\text {sat }}\left(T_{0}\right)-p_{g}\right) \nu_{l \rightarrow g}
$$


where $p^{\text {sat }}$ denotes the saturation pressure of the considered chemical species.

The influence of the liquid-vapor interface on the domain boundary condi-

\subsubsection{Averaged phase change term}

This approach is motivated by the consistency of the averaged model, in the sense that the averaged model should converge to the exact model when the size of the averaging volume goes to zero. A lack of consistency in an averaged

220 model is not necessarily a problem. For a complex flow (as for instance in [10], [11] or [36]), the resolution of the computation will never be increased to

They are the balance laws for gas mass, gas momentum and gas total energy [24]. The corresponding equations for the liquid can be derived by subtracting (6) from (2).

\subsection{Averaging}

Equations (6) will now be averaged for numerical resolution in an Eulerian finite volume framework. The reader can refer to [11] for a complete study on the derivation of such an averaged model. In this paper, we will focus on the phase change terms of the form $J \cdot \nabla \chi$, for which a different approach to [11] will be proposed. 
capture scales at which the phases are separated. It is thus more important to ensure that the chosen approximations are experimentally valid at large scale rather than asymptotically consistent. However, this is not the case when the averaged model is aimed at the description of a cell (or a few cells) of a liquidvapor interface between two separated fluids, such as in [21], [22] or [28]. In these references, as well as in our work, the mixture is a numerical bias at the interface. Several numerical techniques can be used to limit the size of the mixture layer, but this size is never perfectly controlled. Our objective is that phase change should be as independent as possible of the thickness of the numerical mixture layer at the interface, and that consistency with the exact model (6) is maintained when the thickness goes to zero.

The averaging operator will be denoted by ${ }^{-}$in the following paragraphs. In the usual approach, as seen in e.g. [11], the phase change term is averaged as

$$
\overline{J \cdot \nabla \chi} \simeq \overline{J_{l \rightarrow g}} \mathcal{A}_{i},
$$

${ }^{235}$ where $\overline{J_{l \rightarrow g}}$ is a local average interfacial evaporation mass flux and $\mathcal{A}_{i}$ is a local interfacial area density, which is typically a function of the gas volume fraction $\alpha_{g}=\bar{\chi}$.

The resulting phase change term is of order 0 (i.e. it does not contain derivatives of the state variables). The interface evolves in a similar way to a 240 reaction-diffusion equation. It is possible to check if the total exchanged mass is strongly influenced by the thickness of the mixture layer. Moreover, it is not an easy task to write a reaction-diffusion equation which converges asymptotically towards a discontinuous wave front (see e.g. [37, 38]).

Besides, let us note that the "standard" averaged model without phase change 245 (see e.g. [31]) is consistent. The term $p \nabla \chi$ in (6b), that can be seen as an interfacial condition between the two fluids (pressure force of one upon the other), is usually averaged as $p \nabla \alpha_{g}$ (plus other source terms), which naturally degenerates into $p \nabla \chi$.

The interfacial phase change is also described in (2) and (6) as a first order 
term. To take an average in a consistent way, it seems reasonable to use a first order term. We will follow the example of $p \nabla \chi$ and average the term $J \cdot \nabla \chi$ under the following form

$$
\overline{J \cdot \nabla \chi} \simeq \bar{J} \cdot \nabla \alpha_{g} .
$$

As for the pressure term, additional source terms could have been added to the approximation, but since it is assumed that these terms become negligible when the averaging volume goes to 0 (that is $\alpha_{g} \longrightarrow 0$ or 1 ), they will not be considered here. In the remainder of this paper, the notation is simplified by using $J$ instead of $\bar{J}$.

\subsubsection{Average balance equations}

In this paragraph, the usual notation is used for two phase flows $\left(\alpha:=\alpha_{g}\right.$ 260 is the void fraction, $\rho_{g}$ and $\rho_{l}$ are the densities of the gas and liquid phases, $u_{g}$ and $u_{l}$ their velocities, $E_{g}$ and $E_{l}$ their total specific energies) and the following standard approximations are made

$$
\begin{aligned}
\overline{\chi(\rho u \otimes u+p)} & \simeq \alpha_{g}\left(\rho_{g} u_{g} \otimes u_{g}+p_{g}\right), \\
\overline{\chi((\rho E+p) u+q)} & \simeq \alpha_{g}\left(\left(\rho_{g} E_{g}+p_{g}\right) u_{g}+q_{g}\right),
\end{aligned}
$$

where $p_{g}$ is the gas pressure, given by the equation of state $p_{g}=p\left(\rho_{g}, e_{g}\right)$, and similarly for the liquid phase.

After averaging of equations (6) and their counterparts for the liquid phase, and neglecting the diffusive and source terms, the following six equations system is found:

$$
\begin{gathered}
\partial_{t}\left(\alpha_{k} \rho_{k}\right)+\nabla \cdot\left(\alpha_{k} \rho_{k} u_{k}\right)+J \cdot \nabla \alpha_{k}=0, \\
\partial_{t}\left(\alpha_{k} \rho_{k} u_{k}\right)+\nabla \cdot\left(\alpha_{k}\left(\rho_{k} u_{k} \otimes u_{k}+p_{k} \mathbb{I}\right)\right)-P \nabla \alpha_{k}=0, \\
\partial_{t}\left(\alpha_{k} \rho_{k} E_{k}\right)+\nabla \cdot\left(\alpha_{k}\left(\left(\rho_{k} E_{k}+p_{k}\right) u_{k}+q_{k}\right)\right)-Q \cdot \nabla \alpha_{k}=0,
\end{gathered}
$$

for $k=l$ or $g$. The momentum flux (or stress tensor) $P$ across the interface between two phases is $P \nabla \alpha_{g}=\overline{(u \otimes J+p \mathbb{I}) \nabla \chi}$, and the energy flux $Q$ is defined as $Q \cdot \nabla \alpha_{g}=\overline{(J E+p u+q) \cdot \nabla \chi}$. 


\subsubsection{Closure} $\left.u_{g}, E_{g}, p_{g}, q_{g}, \alpha_{l}, \rho_{l}, u_{l}, E_{l}, p_{l}, q_{l}, J_{l \rightarrow g}, P \nabla \alpha, Q \cdot \nabla \alpha\right)$.

The system (7) to which $\alpha_{g}+\alpha_{l}=1$, two equations of state and the Fourier laws in both phases are added, contains in total $7+4 n$ equations. Hence $2+2 n$ closure relations are missing.

The mass and energy fluxes can be related to the local thermodynamic states at the interface (see [24]) in the form:

$$
J=\mathcal{J}_{l \rightarrow g}\left(p_{g}, T_{g}, p_{l}, T_{l}\right) \nu_{l \rightarrow g}, \quad Q=\mathcal{Q}_{l \rightarrow g}\left(p_{g}, T_{g}, p_{l}, T_{l}\right) \nu_{l \rightarrow g}
$$

The following closure relation for $P$ can be added:

$$
P \nabla \alpha=\left(u_{g} \otimes J+p_{g} \mathbb{I}\right) \nabla \alpha=\left(u_{l} \otimes J+p_{l} \mathbb{I}\right) \nabla \alpha
$$

which is a generalization of the single-pressure closure $p_{g}=p_{l}$. This expression for $P \nabla \alpha$ ensures the consistency of the averaged model.

\subsection{Single-velocity hypothesis}

The averaged model described in the previous paragraphs will not be studied in more depth in this paper. Instead, a simplified model derived from it will be discussed.

\subsubsection{Derivation}

The single-pressure and single-velocity hypotheses $\left(p_{g}=p_{l}\right.$ and $\left.u_{g}=u_{l}\right)$ are often used for modelling flows with two separated phases when the numerical method involves mixture cells for the description of the interface (as for instance in [21] or [22]). These hypotheses are consistent with the boundary conditions around an interface without phase change. However, at an interface with phase change this is no longer true.

Indeed, at a liquid-vapor interface with phase change, the flow respects the following jump conditions (see e.g. [34]):

$$
p_{g}-p_{l}=J \cdot\left(u_{l}-u_{g}\right)
$$




$$
\left(u_{g}-u_{l}\right) \cdot \nu_{l \rightarrow g}=J\left(\frac{1}{\rho_{g}}-\frac{1}{\rho_{l}}\right) .
$$

The velocity jump in (10) is due to the difference in densities: a change in across the interface. The pressure jump in (9) can be seen as the corresponding Venturi effect.

The usual single-velocity and single-pressure hypotheses are replaced with relations (9) and (10). This is completed with the following standard condition for the tangential velocities:

$$
u_{g} \cdot \nu^{\perp}=u_{l} \cdot \nu^{\perp},
$$

for any tangential vector $\nu^{\perp}$ at the interface.

These simplifying hypotheses will be applied to the following isothermal system directly derived from (7) and (8):

$$
\begin{aligned}
\partial_{t}\left(\alpha_{k} \rho_{k}\right) & +\nabla \cdot\left(\alpha_{k} \rho_{k} u_{k}\right)-J \cdot \nabla \alpha_{k}=0, \\
\partial_{t}\left(\alpha_{k} \rho_{k} u_{k}\right) & +\nabla \cdot\left(\alpha_{k}\left(\rho_{k} u_{k} \otimes u_{k}+p_{k} \mathbb{I}\right)\right)-\left(u_{k} \otimes J+p_{k} \mathbb{I}\right) \nabla \alpha_{k}=0,
\end{aligned}
$$

for $k=l, g$.

The averaged quantities $\rho_{m}$ and $u_{m}$ are defined such that:

$$
\rho_{m}=\sum \alpha_{k} \rho_{k}, \quad \rho_{m} u_{m}=\sum \alpha_{k} \rho_{k} u_{k} .
$$

Using this notation, the single-velocity hypothesis (10) can be rewritten as

$$
u_{m}-\frac{J}{\rho_{m}}=u_{k}-\frac{J}{\rho_{k}} .
$$

305

Mass fraction $\xi$ is defined as $\xi_{k} \rho_{m}=\alpha_{k} \rho_{k}$. for $k=l, g$ and from definition of $\rho_{m}, \xi_{g}+\xi_{l}=1$.

Finally, the two mean pressures $\bar{p}$ and $p$ are introduced as

$$
\underline{p}=\sum \alpha_{k} p_{k}, \quad \bar{p}=\sum \xi_{k} p_{k} .
$$

For simplicity, the following notation is used for the rest of the article:

$$
\xi:=\xi_{g}, \quad \alpha:=\alpha_{g} .
$$


Using this notation and hypotheses (9), (10) and (11), system (12) can be rewritten as [24]

$$
\begin{aligned}
\partial_{t} \rho_{m} & +\nabla \cdot\left(\rho_{m} u_{m}\right)=0, \\
\partial_{t} \rho_{m} u_{m} & +\nabla \cdot\left(\rho_{m} u_{m} \otimes u_{m}+\underline{p} \mathbb{I}+(\bar{p}-\underline{p}) \nu_{l \rightarrow g} \otimes \nu_{l \rightarrow g}\right)=0, \\
\partial_{t} \xi \rho_{m}+\nabla \cdot\left(\xi \rho_{m} u_{m}+J(\alpha-\xi)\right)-J \cdot \nabla \alpha=0 . &
\end{aligned}
$$

In $1 \mathrm{D}$, this system becomes

$$
\begin{gathered}
\partial_{t} \rho_{m}+\partial_{x}\left(\rho_{m} u_{m}\right)=0, \\
\partial_{t} \rho_{m} u_{m}+\partial_{x}\left(\rho_{m} u_{m}^{2}+\bar{p}\right)=0, \\
\partial_{t} \xi \rho_{m}+\partial_{x}\left(\xi \rho_{m} u_{m}+J(\alpha-\xi)\right)-J \cdot \partial_{x} \alpha=0 .
\end{gathered}
$$

\subsubsection{Simplification and summary}

form its resolution (in particular in 2D or 3D) would be difficult. Therefore, some simplifications will be made.

The hypotheses (9) and (10) can be written as

$$
u_{g}-u_{l}=O(J), \quad p_{g}-p_{l}=O\left(J^{2}\right) .
$$

For small mass flux $J$ the pressure difference at the interface is neglected. Moreover the following assumption is made:

$$
\nabla(J(\alpha-\xi))-J \cdot \nabla \alpha \simeq-J \cdot \nabla \xi .
$$

Hence, the simplified system becomes:

$$
\begin{aligned}
& \partial_{t} \rho_{m}+\nabla \cdot\left(\rho_{m} u_{m}\right)=0, \\
& \partial_{t}\left(\rho_{m} u_{m}\right)+\nabla \cdot\left(\rho_{m} u_{m} \otimes u_{m}+p \mathbb{I}\right)=0, \\
& \partial_{t}\left(\xi \rho_{m}\right)+\nabla \cdot\left(\xi \rho_{m} u_{m}\right)-J \cdot \nabla \xi=0 .
\end{aligned}
$$

315

Only system (16) will be used to compute the test cases that follow. This averaged system is conveniently similar to the isothermal version of the original 
system (2), and so the discussion on the hyperbolicity of the isothermal system and its solution to the Riemann problem are still valid.

System (16) is completed by the following closure relations:

$$
\rho_{g}=\mathcal{R}_{g}(p), \quad \rho_{l}=\mathcal{R}_{l}(p), \quad \alpha=\frac{\xi \rho_{m}}{\rho_{g}}, \quad \rho_{m}=\alpha \rho_{g}+(1-\alpha) \rho_{l},
$$

where functions $\mathcal{R}_{g}$ and $\mathcal{R}_{l}$ are isothermal equations of state for the two phases. When the phase change kinetics in (5) are included, the system contains $6+2 n$ equations for $6+2 n$ variables $\left(\rho_{m}, u_{m}, p, \xi, \rho_{g}, \rho_{l}, \alpha, J\right)$.

The variables $\rho_{m}, u_{m}$ and $\xi$ are the averaged equivalents of the exact variables $\rho, u$ and $\chi$. The averaged versions are expected to converge to their exact counterparts when the size of the averaging volume goes to zero. In the rest of this paper, only the averaged quantities will be considered and the $m$ index will be dropped for the sake of readability.

Note that two averaged versions of the order parameter $\chi$ have actually been introduced: the mass fraction $\xi$ and the volume fraction $\alpha$. Due to the density ratio, both values can be fairly different (as seen for example in Figure 4 below). Some of the difficulties of this model come from this difference as we will see in Section 4

\section{Discretization}

In this section the discretization of the system (16) will be discussed.

\subsection{Discretization of the non-conservative terms}

The model (16) involves a non-conservative term of the form $J \cdot \nabla \xi$. The discretization strategy is to approximate the term locally using a conservative term. This approach is inspired by (but different from) the strategy of [31]. This approximation is presented in the general case of a system with non-conservative terms of the form

$$
\partial_{t} v+\nabla \cdot F(v)+C(v): \nabla v=0
$$




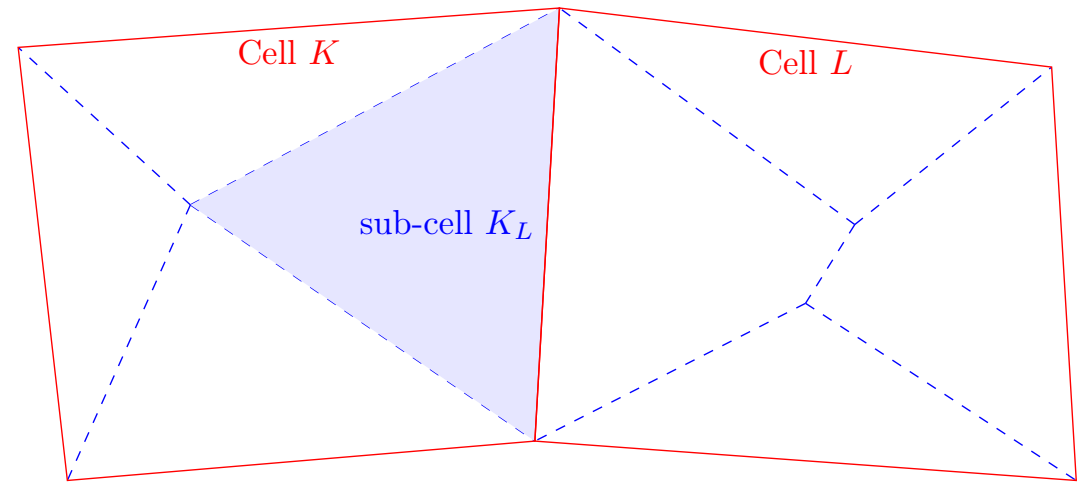

Figure 1: Example decomposition of the cells (solid red lines) into sub-cells (dashed blue lines) for the integration of $2 \mathrm{D}$ non-conservative terms.

where $C(v)$ is a tensor and : denotes the tensor-matrix product such that the result is a vector. However in this paper only our simple phase change term will be tested.

Each cell will be decomposed into sub-cells for the linearization of the nonconservative terms. The final scheme does not depend on the chosen decomposition, as it is only a technical tool for the derivation of the discretized equation.

Let $K$ be a cell with its neighbors denoted by $\mathcal{N}(K)$. Cell $K$ is divided into $\# \mathcal{N}(K)$ sub-cells $\left\{K_{L}\right\}_{L \in \mathcal{N}(K)}$, such that $K_{L}$ is only in contact with the neighboring cell $L$ (see Figure 1 for an example). If $K$ is a convex polygon (or polyhedron), such a partition of $K$ is easily achieved by joining the center of mass of the cell to each of its corners.

The existence of a flux function $G$ is assumed on the two neighboring subcells, which approximates the non-conservative term around each face, in the following way:

$$
\forall x \in K_{L} \cup L_{K}, \quad C(v): \nabla v \simeq \nabla \cdot G_{K, L}(v) .
$$

In the spirit of [31] we could have taken

$$
G_{K, L}(v)=C\left(\mu_{K, L}\right) A^{-1}\left(\mu_{K, L}\right) F(v)
$$


where $\mu_{K, L}$ is an average state between $v_{K}$ and $v_{L}$ and

$$
A(v)=\frac{\partial F}{\partial v} .
$$

For our phase change model (16), we will instead choose the simpler

$$
\forall x \in K_{L} \cup L_{K}, \quad J \cdot \nabla \xi \simeq \nabla \cdot\left(\mathcal{J}_{l \rightarrow g}\left(v_{K}, v_{L}\right)\left(\nu_{l \rightarrow g}\right)_{K, L} \xi\right),
$$

where $\mathcal{J}_{l \rightarrow g}$ is the physical evaporation mass flux as defined in (5) and $\left(\nu_{l \rightarrow g}\right)_{K, L}$ is the normal vector to the liquid-vapor interface as approximated at the mesh face.

Using the approximation (19), the spatial derivative of the partial differential equation system can be integrated as follows [24]:

$$
\int_{K}(\nabla \cdot F+C: \nabla v) \mathrm{d} x \simeq \sum_{L \in \mathcal{N}(K)}|\partial K \cap \partial L|\left[\Phi_{K, L}-G_{K, L}\left(v_{K}\right)\right] \cdot \nu_{K, L},
$$

where $\Phi_{K, L}$ denotes the numerical flux approximating $F+G_{K, L}$ at the interface between $K$ and $L,|\partial K \cap \partial L|$ is the area of the interface and $\nu_{K, L}$ is its normal unit vector.

\subsection{Numerical flux}

Let us now discuss the approximation of the flux at the interface. The discretization proposed below is based on the Roe-type scheme of [31].

In the previous section it has been seen how an equation is locally approximated with non-conservative terms as a conservation equation. Let us thus consider a conservation equation of the form

$$
\partial_{t} v+\partial_{x} F(v)=0
$$

Multiplying this equation by $A(v)=\frac{\partial F}{\partial v}$, we get

$$
\partial_{t} F(v)+A(v) \partial_{x} F(v)=0 .
$$

At an interface between cells $K$ and $L$ this equation is approximated in the form

$$
\partial_{t} F(v)+\widetilde{A}\left(v_{K}, v_{L}\right) \partial_{x} F(v)=0,
$$


where the matrix $\widetilde{A}$ is such that

$$
\widetilde{A}(v, v)=A(v) .
$$

Equation (24) is linear, so it is easily solved exactly to find the flux between the cells.

This approach is similar to the Roe scheme, although we aim to avoid the need for a Roe matrix condition $\widetilde{A}\left(v_{K}, v_{L}\right) \cdot\left(v_{K}-v_{L}\right)=\left(F_{K}-F_{L}\right)$ by linearizing зво (23) instead of (22) (see also [31]).

In [31] an average state $\mu_{K, L}$ at the interface is used to define $\widetilde{A}$. At a liquid-vapor interface the definition of an intermediate state between liquid and gas might be problematic (for instance, it is not clear how to define the equation of state of this average state). Thus below, a matrix $\widetilde{A}$ is defined, which depends minimally on any intermediate state. This choice will be validated by demonstrating good properties of the resulting scheme (see Section 3.3).

We denote $\lambda_{k}$ as the $k$-th eigenvalue of $A, l_{k}$ as an associated left eigenvector and $L=\left(l_{k}\right)_{1 \leq k \leq n}$ as a matrix of left eigenvectors, such that $A=$ $L^{-1} \operatorname{diag}\left(\lambda_{k}\right) L$.

390

We call "upwind-l" Finite Volume with Characteristic Flux (FVCF) scheme the finite volume scheme which uses the following numerical flux on the face between cells $K$ and $L$ in the direction of the unit normal vector $\nu_{K, L}$ :

$$
\phi_{K, L}=\frac{F\left(v_{K}\right)+F\left(v_{L}\right)}{2} \cdot \nu_{K, L}+\operatorname{sgn} \widetilde{A}\left(v_{K}, v_{L}\right)\left(\frac{F\left(v_{K}\right)-F\left(v_{L}\right)}{2} \cdot \nu_{K, L}\right),
$$

where

$$
\operatorname{sgn} \widetilde{A}\left(v_{K}, v_{L}\right)=\widetilde{L}\left(v_{K}, v_{L}\right)^{-1} \operatorname{diag}\left(\operatorname{sgn}\left(\lambda_{k}\left(\mu_{K, L}\right)\right)\right) \widetilde{L}\left(v_{K}, v_{L}\right) .
$$

For all $k$, the $k$-th line of the eigenvector matrix $\widetilde{L}$ is defined by

$$
\left(\widetilde{L}\left(v_{K}, v_{L}\right)\right)_{k}= \begin{cases}l_{k}\left(v_{K}\right) & \text { if } \lambda_{k}\left(\mu_{K, L}\right)>0 \\ l_{k}\left(v_{L}\right) & \text { if } \lambda_{k}\left(\mu_{K, L}\right)<0 \\ l_{k}\left(\mu_{K, L}\right) & \text { else }\end{cases}
$$


and $\mu_{K, L}$ is an average state between $v_{K}$ and $v_{L}$.

This numerical flux corresponds to the following upwind resolution of (24):

$$
\begin{cases}l_{k}\left(v_{K}\right) \phi_{K, L}=l_{k}\left(v_{K}\right)\left(F\left(v_{K}\right) \cdot \nu_{K, L}\right) & \text { if } \lambda_{k}\left(\mu_{K, L}\right)>0 \\ l_{k}\left(v_{L}\right) \phi_{K, L}=l_{k}\left(v_{L}\right)\left(F\left(v_{L}\right) \cdot \nu_{K, L}\right) & \text { if } \lambda_{k}\left(\mu_{K, L}\right)<0 .\end{cases}
$$

The case when $\lambda_{k}\left(\mu_{K, L}\right)=0$ has not really been studied in this work, but it is expected to be unimportant in most simulations.

The reader is referred to [35] for a discussion on the discretization of the boundary conditions with this kind of scheme.

The application of numerical flux (25) to the linearized non-conservative system described in Section 3.1 gives

$$
\begin{aligned}
\Phi_{K, L}= & \frac{\left(F+G_{K, L}\right)\left(v_{K}\right)+\left(F+G_{K, L}\right)\left(v_{L}\right)}{2} \cdot \nu_{K, L} \\
& +\operatorname{sgn}\left[\widetilde{\boldsymbol{A}_{K, L}}\left(v_{K}, v_{L}\right)\right] \frac{\left(F+G_{K, L}\right)\left(v_{K}\right)-\left(F+G_{K, L}\right)\left(v_{L}\right)}{2} \cdot \nu_{K, L},
\end{aligned}
$$

where

$$
\boldsymbol{A}_{K, L}(v)=\frac{\partial\left(F+G_{K, L}\right)}{\partial v}
$$

\subsection{Maximum principle}

As mentioned previously, this scheme has good properties with respect to the maximum principle of the variable $\xi$ as studied for instance in [39]. For the sake of clarity, only the $1 \mathrm{D}$ case will be presented here.

Assuming

$$
\max \left(\Phi_{j+1 / 2}^{\rho}-J_{j+1 / 2}, 0\right)-\min \left(\Phi_{j-1 / 2}^{\rho}-J_{j-1 / 2}, 0\right)-J_{j-1 / 2}+J_{j+1 / 2} \leq \rho_{j} \frac{\Delta x}{\Delta t}
$$

and

$$
\forall j, \quad \operatorname{sgn}\left[\left(u-\frac{J}{\rho}\right)\left(\mu_{j+1 / 2}\right)\right]=\operatorname{sgn}\left[\Phi_{j+1 / 2}^{\rho}-J_{j+1 / 2}\right],
$$

this scheme respects the local discrete maximum principle of $\xi$, that is

$$
\forall j, \quad \forall n, \quad \min \left(\xi_{j-1}^{n}, \xi_{j}^{n}, \xi_{j+1}^{n}\right) \leq \xi_{j}^{n+1} \leq \max \left(\xi_{j-1}^{n}, \xi_{j}^{n}, \xi_{j+1}^{n}\right)
$$


The first condition (28a) is a CFL-like condition on $u-J / \rho$. In most practical cases, this should be less restrictive than the CFL condition for $u \pm c$, where $c$ is the speed of sound.

The second condition (28b) is an implicit condition on $\operatorname{sgn}\left(\lambda_{2}\left(\mu_{j+1 / 2}\right)\right)$.

415 For the "upwind-l" FVCF scheme, this is the only role played by the averaged state $\mu_{j+1 / 2}$. Using the velocity of the acoustic solver

$$
u_{j+1 / 2}=\frac{\rho_{j} c_{j} u_{j}+\rho_{j+1} c_{j+1} u_{j+1}}{\rho_{j} c_{j}+\rho_{j+1} c_{j+1}}+\frac{p_{j}-p_{j+1}}{\rho_{j} c_{j}+\rho_{j+1} c_{j+1}},
$$

as the velocity of the intermediate state $\mu_{j+1 / 2}$ is sufficient in most practical cases.

Equation (29) is important because it ensures that non-physical mass fractions, $\xi<0$ or $\xi>1$, do not appear. For instance, the numerical scheme of [20] does not respect this property and non-physical mass fractions can appear, even for simple 1D advection problems without phase change. These non-physical values often cause the simulation to fail. The development of the new variant of the FVCF scheme presented in the previous paragraphs was motivated by this 425 property.

\section{Validation test cases}

Some simple test cases are presented in this section that aim to validate the model and its implementation. In particular, one of our objectives is to create a model for which phase change is as independent as possible of the thickness of the numerical mixture layer at the interface. However, no reference test case for this kind of model was found in the literature, thus original test cases will be proposed.

\section{1. $1 D$ forced oscillating phase change}

The purpose of this first test case is to validate the ability of the code to describe the interface and deal with non-trivial density ratios. In order to focus on these points, the mass flux will be independent of the system evolution. Using 
this hypothesis, an analytical solution will be provided for comparison with the numerical results.

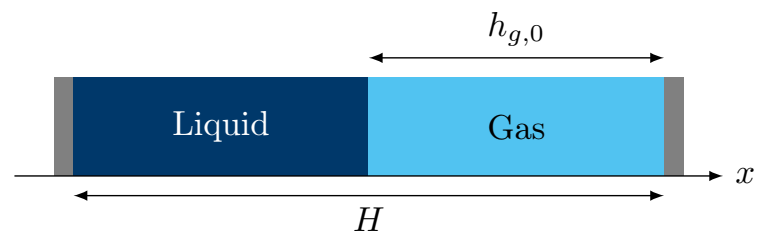

Figure 2: Initial conditions for the test cases given in Sections 4.1 and 4.3.

Considering a tube of section $S$ and length $H$ that is closed at both ends.

440 The tube is initially filled with a volume $S \times h_{g, 0}$ of gas and $S \times\left(H-h_{g, 0}\right)$ of liquid (see Figure 2). These phases respectively follow the isothermal ideal gas and isothermal stiffened gas equations of state, that is:

$$
\rho_{g}(p)=\frac{p}{c_{g, 0}^{2}}, \quad \rho_{l}(p)=\rho_{l, 0}+\frac{p-p_{0}}{c_{l, 0}^{2}},
$$

where $c_{g, 0}$ and $c_{l, 0}$ are the respective sound speeds in the gas and liquid. We denote by $\rho_{g, 0}$ and $\rho_{l, 0}$ the densities at the reference pressure $p_{0}$ (that is $p_{0}=$ $\left.{ }_{445} \rho_{g, 0} c_{g, 0}^{2}\right)$.

\subsubsection{Choice of $J_{l \rightarrow g}$}

An evaporation mass flux $J_{l \rightarrow g}(t)$ is set at the interface to be $J_{l \rightarrow g}(t)=$ $A \sin (\omega t)$ with constant coefficients $A$ and $\omega$. The choice of $A$ and $\omega$ is governed by the following constraints:

- The pressure must remain positive (and thus the densities also remain positive). For this, it is sufficient to set

$$
A>0 .
$$

- The fluid velocity must be subsonic $\forall t, u(t)<c$, with the following upper bound for the escaping gas velocity

$$
\max \left(u_{g}\right) \leq \frac{\max \left(\left|J_{l \rightarrow g}\right|\right)}{\min \left(\rho_{g}\right)}=\frac{A}{\rho_{g, 0}}<c_{g, 0} .
$$


Note that this upper bound is actually never reached during the simulation $\rho_{g}$.

- In order to compare with the analytical solution, the variations in mass flux must be slow enough for the homogeneous pressure hypotheses to be valid:

$$
\omega \ll \frac{c_{g, 0}}{H}
$$

460

\subsubsection{Dimensionless form}

The previous equations can be rewritten in dimensionless form. Dimensionless variables are denoted with a tilde and they are defined as

$$
\begin{gathered}
h_{g}=h_{g, 0} \widetilde{h_{g}}, \quad \rho_{g}=\rho_{g, 0} \widetilde{\rho_{g}}, \quad \rho_{l}=\rho_{g, 0} \widetilde{\rho_{l}}, \quad t=\frac{h_{g, 0}}{c_{g, 0}} \widetilde{t}, \\
p=p_{0} \widetilde{p}, \quad J_{l \rightarrow g}=\rho_{g, 0} c_{g, 0} \widetilde{J}_{l \rightarrow g} .
\end{gathered}
$$

The equations of state then read:

$$
\widetilde{\rho_{g}}=\widetilde{p}, \quad \widetilde{\rho_{l}}=\frac{1+\mathrm{CR}(\widetilde{p}-1)}{\mathrm{DR}} .
$$

465

where DR and CR are respectively the density ratio and compressibility ratio, defined as follows:

$$
\mathrm{DR}=\frac{\rho_{g, 0}}{\rho_{l, 0}}, \quad \mathrm{CR}=\frac{\rho_{g, 0} c_{g, 0}^{2}}{p_{0}} \times \frac{p_{0}}{\rho_{l, 0} c_{l, 0}^{2}}=\frac{\rho_{g, 0} c_{g, 0}^{2}}{\rho_{l, 0} c_{l, 0}^{2}} .
$$

The total length of the domain is written in dimensionless form as the aspect ratio $\widetilde{H}$

$$
\widetilde{H}=\frac{H}{h_{g, 0}} .
$$

The mass flux can be written as

$$
\widetilde{J}_{l \rightarrow g}=\operatorname{Ma} \sin (\widetilde{\omega} \widetilde{t}),
$$

470 where

$$
\widetilde{\omega}=\frac{\omega h_{g, 0}}{c_{g, 0}}, \quad \mathrm{Ma}=\frac{A}{\rho_{g, 0} c_{g, 0}} .
$$


The dimensionless number Ma can be understood as the Mach number associated with the maximal escaping gas velocity in equation (30b).

The initial conditions can be written in dimensionless form as

$$
\widetilde{p}(0, \widetilde{x})=1, \quad \begin{cases}\xi(0, \widetilde{x})=1 & \text { if } 0<\widetilde{x}<1, \\ \xi(0, \widetilde{x})=0 & \text { if } 1<\widetilde{x}<\widetilde{H}\end{cases}
$$

where the dimensionless abscissa $\widetilde{x}$ is defined as $h_{g, 0} \widetilde{x}=x$.

Finally, conditions (30) become

$$
0<\mathrm{Ma}<1, \quad \widetilde{\omega} \widetilde{H} \ll 1 .
$$

The problem depends on five parameters, which are DR, CR, $\widetilde{H}$, Ma and $\widetilde{\omega}$. Table 1 proposes some values for these parameters, where the values of DR and $\mathrm{CR}$ are inspired by the properties of water and steam.

\begin{tabular}{c|c}
$\mathrm{DR}$ & $10^{-3}$ \\
$\mathrm{CR}$ & $4 \cdot 10^{-5}$ \\
$\widetilde{H}$ & 2 \\
$\mathrm{Ma}$ & 0.1 \\
$\widetilde{\omega}$ & 0.05
\end{tabular}

Table 1: Dimensionless parameters used for the numerical resolution.

\subsubsection{Analytical solution}

Assuming a homogeneous pressure in the tube, the dimensionless pressure is the solution of: [24]

$$
(1+\mathrm{CR}(\widetilde{p}-1))\left(\widetilde{H}-\frac{1+\widehat{m}(t)}{\widetilde{p}}\right)-\widetilde{H}+1+\operatorname{DR} \widehat{m}(t)=0
$$

where $\widehat{m}(t)$ is the dimensionless exchanged mass:

$$
\widehat{m}(t)=\int_{0}^{\widetilde{t}} \widetilde{J}_{l \rightarrow g}(s) \mathrm{d} s .
$$

In practice, the role of liquid compressibility is negligible for the studied fluids. Thus, it can be neglected and the dimensionless pressure $\widetilde{p}(t)$ can be 


$$
\widetilde{p}=\frac{1+\widehat{m}(t)}{1+\operatorname{DR} \widehat{m}(t)}+O(\mathrm{CR})
$$

\subsubsection{Results and comments}

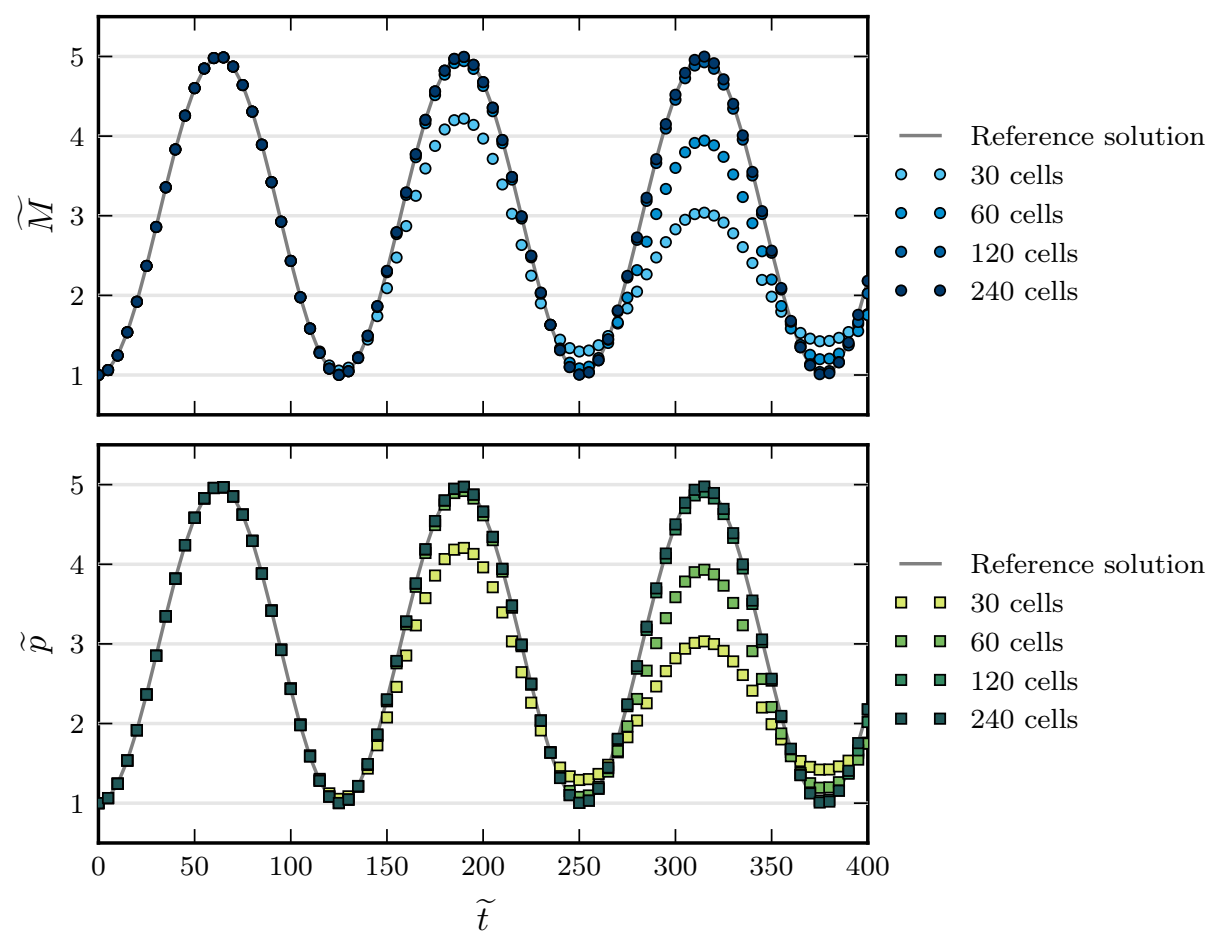

Figure 3: Evolution of dimensionless total mass of gas (top) and dimensionless pressure in the middle of the domain (bottom) as functions of dimensionless time for the numerical simulations and the reference solution. Parameters are given in Table 1 . The period is $2 \pi / \widetilde{\omega} \simeq 125$.

In Figure 3 the time variation of total mass of gas and pressure in the domain is shown. Due to the liquid being very dense and almost incompressible, the volume of gas is nearly constant and the pressure is thus proportional to the total mass of gas (equal in dimensionless magnitude). The numerical results are in good agreement with the exact solution, except for the coarser meshes with 30 and 60 cells.

The inaccuracy of the coarser meshes can be explained by studying the 

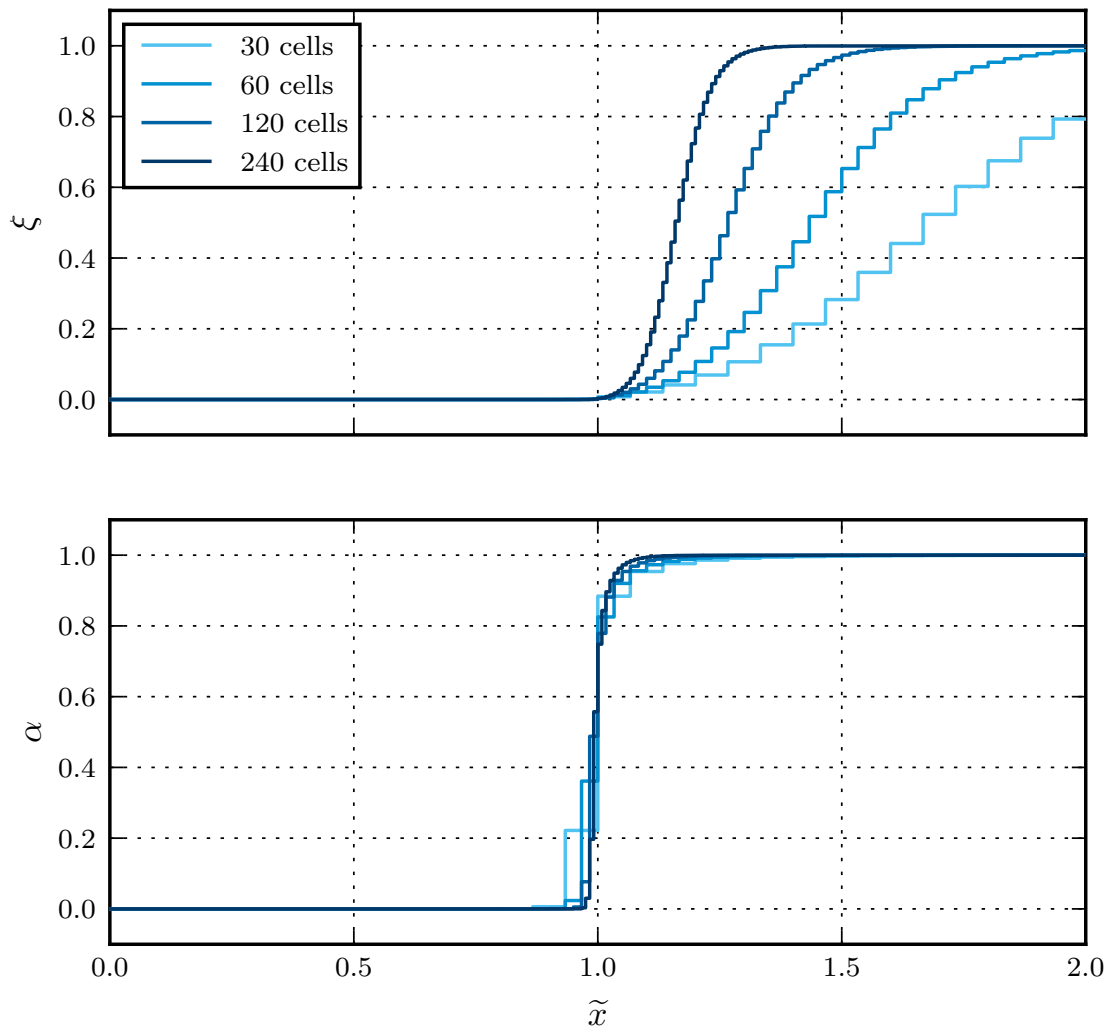

Figure 4: Profiles of mass fraction $\xi$ and of volume fraction $\alpha$ after one period (at $\widetilde{t} \simeq 125$ ), for the same numerical computations as in Figure 3. 
mass fraction profile, shown in Figure 4. Numerical diffusion in the coarser simulations has enlarged the interface, so that it reaches the boundary of the domain. In this situation the total exchanged gas mass is no longer the same $\left(\int|\nabla \xi| \mathrm{d} x \neq 1\right)$. The numerical diffusion is reduced for finer meshes and the bias that occurs due to the interface interacting with the boundary appears later in the computation.

500

Within a single period, the numerical diffusion rate changes with the phase change rate. However, on average over several periods, the thickness of the diffused interface will grow according to $\sqrt{t \Delta x}$. Indeed, for a first order upwind scheme such as ours, the numerical diffusion coefficient is proportional to the cell size $\Delta x$ (see [40] for a derivation of the equivalent equation). Thus, the time taken for the interface to reach the domain boundary will be proportional to the number of cells in the mesh, as seen in Figure 3 between the 30 and 60 cell cases.

Except for this limiting case in which the mixture layer covers the whole gas domain, the numerical solution stays consistent with the analytical solution, despite the change in the thickness of the mixture layer.

The diffusion of the volume fraction $\alpha$ is almost symmetrical around the expected position of the interface. Due to the low density ratio, the profiles of $\xi$ and $\alpha$ appear to be significantly different.

In Figure 5, the velocity profile is shown at a time of maximal evaporation rate. Gas is escaping the interface to the right, which is demonstrated by a positive velocity. The velocity of the liquid is almost zero (the liquid is in fact slightly compressed by the pressure rise). A jump in velocity appears at the interface, as presented in (10). The jump is diffused along the profile in a similar way to $\xi$. A zero velocity is imposed by the boundary condition at the walls, causing a linear velocity gradient in the gas phase.

Finally, the gas velocity profile is shown in Figure 6 . The gas velocity $\widetilde{u_{g}}$ is computed from the mixture velocity $\widetilde{u} \equiv \widetilde{u_{m}}$ with the help of (13). In the diffuse interface (approximately between $\widetilde{x}=1.0$ and $\widetilde{x}=1.3$ ), the gas velocity is not affected by the mixture and is the exact gas velocity at the interface in these 


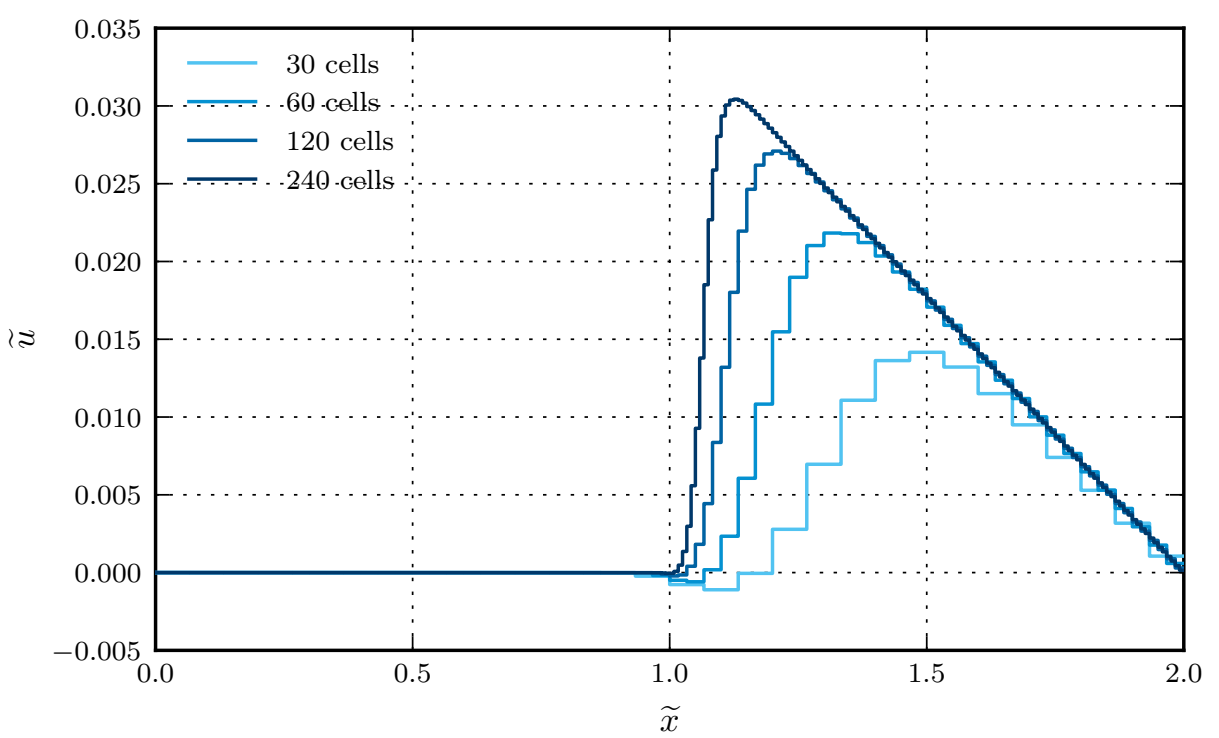

Figure 5: Profiles of dimensionless mixture velocity at $\widetilde{t} \simeq 155$ (approximately $5 / 4$ of a period, at a time when the evaporation mass flux is maximal) for the same numerical computations as in Figure 3. 


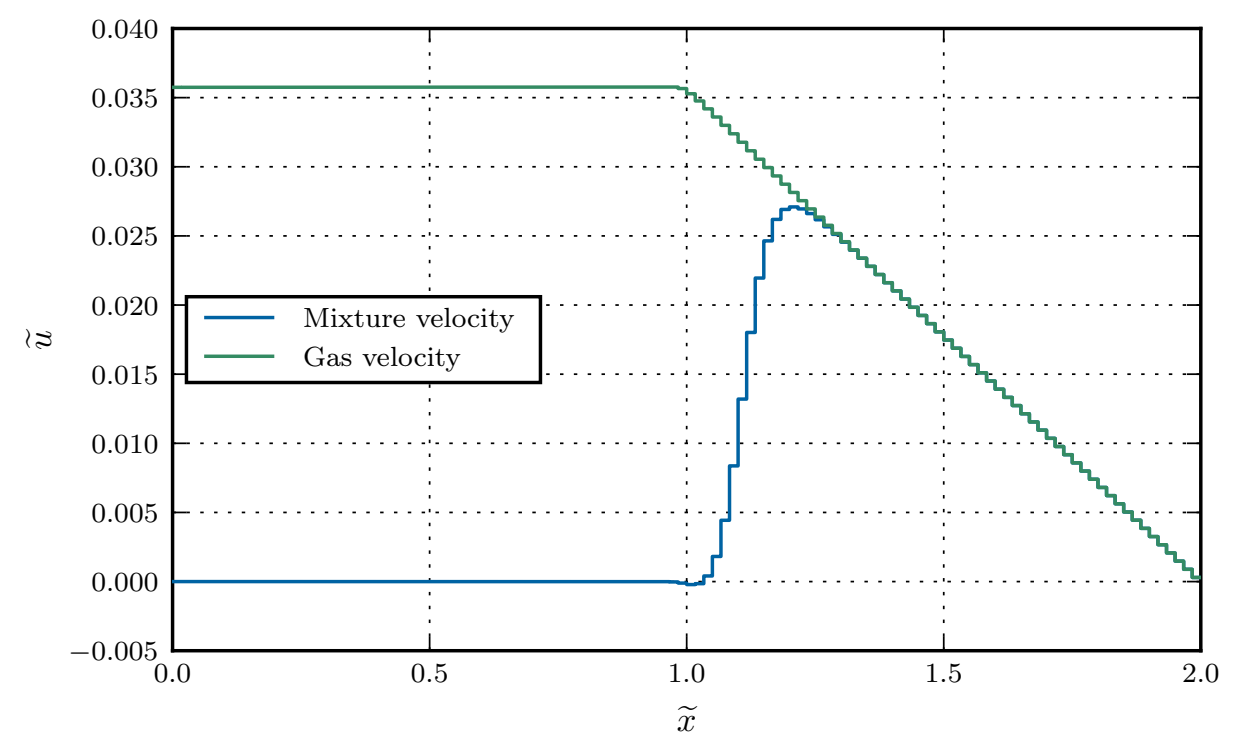

Figure 6: Profiles of dimensionless gas velocity and mixture velocity at $\tilde{t} \simeq 155$ (at $5 / 4$ of a period when the evaporation mass flux is maximal) for the numerical computation with 120 cells. The gas velocity is computed from the mixture velocity using (13). On the left hand side, the mixture is pure liquid and the velocity of the gas is irrelevant. 
conditions. The gas velocity profile converges towards the mixture velocity, as seen in Figure 5, when the diffusion of the interface tends to zero.

\section{2. $2 D$ forced oscillating phase change}

This test case is an extension of the 1D test case in the previous section in two dimensions. Its purpose is to prove the multi-dimensional validity of the model.

\subsubsection{Setup}

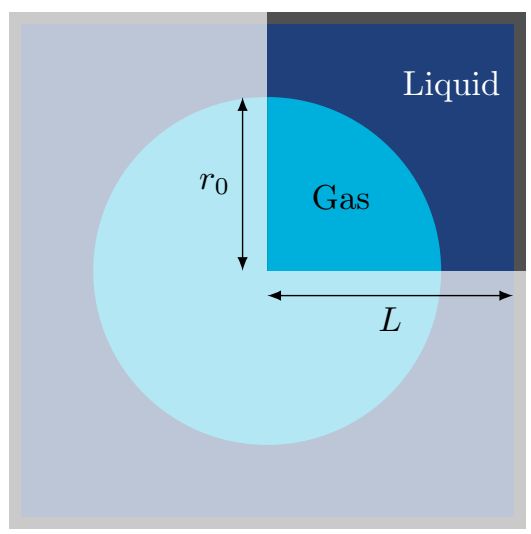

Figure 7: Schematic of the 2D forced phase change test case. By symmetry, only the top right corner will be solved numerically.

Consider a gas bubble of initial radius $r_{0}$ surrounded by liquid (see Figure 7) enclosed in a square domain. As for the previous test case, the fluids are described by the isothermal ideal gas and isothermal stiffened gas equations of state.

Assuming that the pressure is homogeneous in the whole domain and that the liquid is heavy and incompressible (that is $\mathrm{DR} \ll 1$ and $\mathrm{CR} \ll 1$ ), then the pressure satisfies:

$$
p(t)=p_{0}\left(1+\frac{2}{\rho_{g, 0} r_{0}} \int_{0}^{t} J_{l \rightarrow g}(s) \mathrm{d} s\right) .
$$




\subsubsection{Interface normal vector evaluation}

The surfacic mass flux at the interface is given by the same arbitrary expression $J_{l \rightarrow g}(t)=A \sin (\omega t)$ as used in the 1D case. However, in 2D the linearization of the phase change term $J \cdot \nabla \xi$ also requires the computation of the unit normal at the liquid vapor interface $\nu_{l \rightarrow g}$, as seen in (20).

The simplest method is a first order approximation of the following form:

$$
\left(\nu_{l \rightarrow g}\right)_{K, L}= \begin{cases}+\nu_{K, L} & \text { if } \xi_{K}>\xi_{L}, \\ -\nu_{K, L} & \text { else. }\end{cases}
$$

${ }_{545}$ This choice of discretization of $\nu_{l \rightarrow g}$ may reduce the quality of the solution by affecting the total exchanged mass. Namely, the approximation of the interface orientation influences the total length (in 3D the total surface) of the interface. This phenomenon is illustrated in Figure 8. In this case, the total length of the interface is $4 / \pi$ times longer than the actual length. The total exchanged mass will thus be larger for a given surfacic mass flux. To illustrate this phenomenon, the following numerical results are compared to the following variant of (33), which takes this bias into account:

$$
p=p_{0}\left(1+\frac{2}{\rho_{g, 0} r_{0}} \frac{4}{\pi} \int_{0}^{t} J_{l \rightarrow g}(s) \mathrm{d} s\right) .
$$

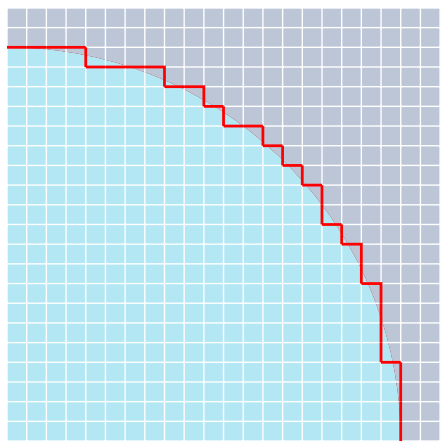

Figure 8: Approximation of an arc circle on a Cartesian mesh. If the radius of the circle is 1, the length of the discrete interface (in red) is always 2 , for any grid resolution. The discrete interface length does not converge towards the actual length of the interface, which is $\pi / 2$. 
The unit normal at the interface may be expressed as $\nu_{l \rightarrow g} \simeq \frac{\nabla \xi}{|\nabla \xi|}$. For a higher order numerical resolution, this can be evaluated with the following formula:

$$
\begin{aligned}
& \frac{\partial \xi}{\partial x} \simeq \frac{\left(\xi_{\mathrm{NE}}-\xi_{\mathrm{NW}}\right)+\left(\xi_{\mathrm{SE}}-\xi_{\mathrm{SW}}\right)}{4 \Delta x} \\
& \frac{\partial \xi}{\partial y} \simeq \frac{\left(\xi_{\mathrm{NW}}-\xi_{\mathrm{SW}}\right)+2\left(\xi_{\mathrm{N}}-\xi_{\mathrm{S}}\right)+\left(\xi_{\mathrm{NE}}-\xi_{\mathrm{SE}}\right)}{4 \Delta x}
\end{aligned}
$$

where the notation is given in Figure 9. The complete study of this kind of discretization (including, for instance, the behavior at the boundary of the domain) is beyond the scope of this paper.

\begin{tabular}{l|c|c}
$\xi_{\mathrm{NW}}$ & $\xi_{\mathrm{N}} \nabla \xi$ & $\xi_{\mathrm{NE}}$ \\
\hline$\xi_{\mathrm{SW}}$ & $\xi_{\mathrm{S}}$ & $\xi_{\mathrm{SE}}$
\end{tabular}

Figure 9: Notation used for the numerical evaluation of the unit normal vector at the liquidvapor interface on a Cartesian mesh as given in (36). The cells are squares of size $\Delta x \times \Delta x$. Here, we wish to linearize the phase change term at the interface between cells $\mathrm{N}$ and $\mathrm{S}$.

Finally, for this particular test case, let us notice that the exact shape of the interface is always known. Due to the problem having spherical symmetry, the normal vector is always given by:

$$
\nu_{l \rightarrow g}=\frac{1}{\sqrt{\left(x-x_{0}\right)^{2}+\left(y-y_{0}\right)^{2}}}\left(\begin{array}{l}
x-x_{0} \\
y-y_{0}
\end{array}\right),
$$

where $\left(x_{0}, y_{0}\right)$ is the center of symmetry of the problem.

These three methods for the computation of $\nu_{l \rightarrow g}$ will be used in the following numerical simulations and their results will be compared.

\subsubsection{Results and comments}

Several computations have been carried out with the same parameters as the $1 \mathrm{D}$ case (see Table 1). Figure 10 displays the evolution of the pressure as a

function of time for various discretizations of the unit normal at the interface. All computations have been made with CFL $=0.25$. 


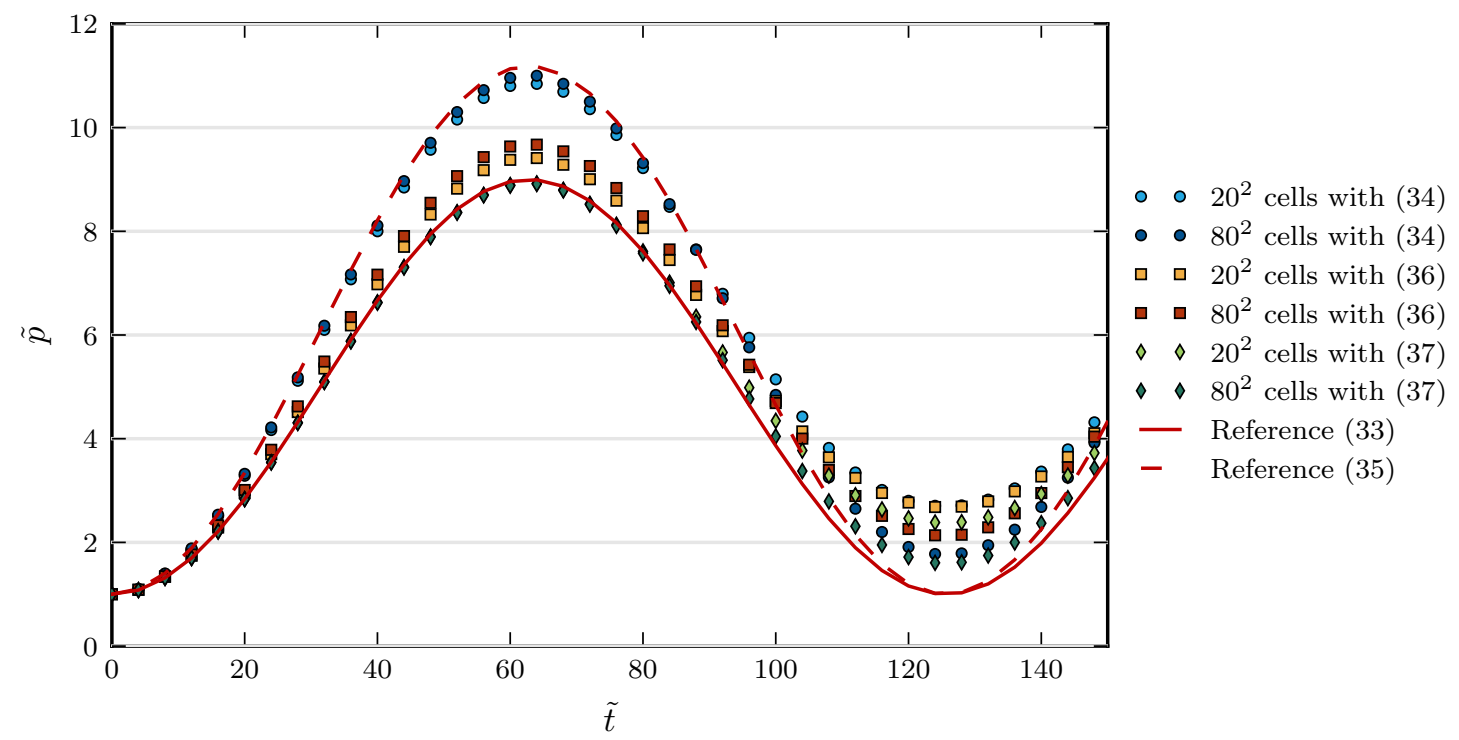

Figure 10: Evolution of the dimensionless pressure (at the center of the domain) as a function of dimensionless time for various mesh sizes and different approximations of the unit normal at the liquid-vapor interface. The plain and dashed red lines are the reference analytical solutions.

As expected, the first order reconstruction of the interface normal (34) is close to the biased analytical solution (35), while the second order reconstruction (36) gives a result that is closer to the exact solution. However, only the exact pressure (33).

In the $1 \mathrm{D}$ case, the effective interfacial area $\int|\nabla \xi| \mathrm{d} x$ remains the same when the interface is diffused (providing the boundaries of the domain are not crossed). So the total mass exchanged between the phases is not affected by numerical diffusion.

The situation is more complex for the $2 \mathrm{D}$ problem because smearing of the liquid-vapor mass fraction distribution actually affects the effective interfacial area. Figure 11 shows the spatial distribution of $\xi$ and $\alpha$ after one oscillation. Due to the density ratio, the gradients of mass fraction $\xi$ and volume fraction 


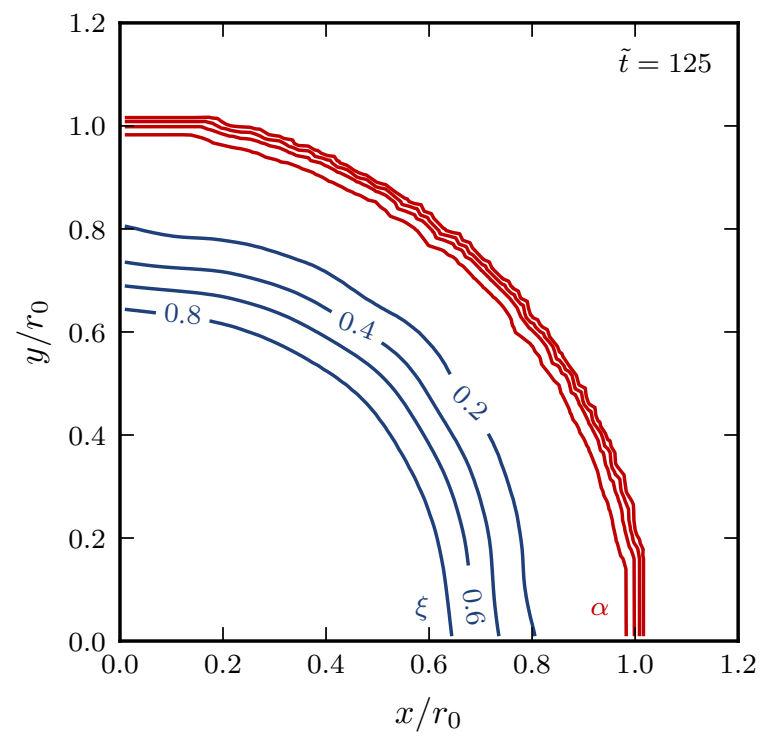

Figure 11: Level set for the mass fraction $\xi$ (in blue) and the volume fraction $\alpha$ (in red) after one period $(\widetilde{t}=125)$ for an $80 \times 80$ cell mesh using the discretization given by $(34)$. To be compared with Figure 4.

$\alpha$ are not superimposed (as in Figure 4). Although the effective interfacial area $\int|\nabla \alpha| \mathrm{d} x$ remains fairly constant, the effective interfacial area $\int|\nabla \xi| \mathrm{d} x$ changes. Since the $\nabla \xi$ term is playing the role of locating the interface in (16), the total quantity of exchanged mass is thus reduced and any pressure oscillations are damped after the first peak.

585

Some good properties of the code have been demonstrated using the 1D test case, particularly regarding the independence of the numerical solution with regard to the thickness of the mixture layer. However, the $2 \mathrm{D}$ case is more difficult and some work should still be done to better capture the solution.

Although $\nabla \xi$ naturally appeared in the derivations of Section 2, checks could be made to show whether its replacement with $\nabla \alpha$ would improve the quality of the model. This replacement is actually a major change in the implementation: the approximation (20) has to be modified and the diagonalization of the advection matrix in the scheme (25) might be significantly affected. In preliminary 
tests, this replacement led to a less smooth and less stable velocity profile at the interface in comparison with Figure 5. The term $\nabla \xi$ appeared in Section 2 as a consequence of the single velocity hypothesis (2.3) and it is thought to be the reason of the good behavior of our code with respect to the velocity jump in $1 \mathrm{D}$.

\section{3. $1 D$ return to equilibrium}

Finally, a simple test case is given that challenges the ability of the code to deal with less trivial expressions for the mass flux.

\subsubsection{Setup}

As mentioned in Section 2.1, the relaxation mass flux will take the following form:

$$
J_{l \rightarrow g}=\rho_{g, 0} c_{g, 0} \Omega \frac{p_{0}-p_{g}}{p_{0}} .
$$

A constant dimensionless relaxation rate $\Omega>0$ is chosen according to similar constraints as (30b). Here, we set $\Omega=0.1$ and $p(t=0)-p_{0} \sim 0.1 p_{0}$, and thus $\max (|J|) \sim 0.01 \rho_{g, 0} c_{g, 0}$. This is the highest relaxation rate that could be simulated for a 120 cell mesh with a CFL number of 0.8 , which corresponds to $\Delta \tilde{t} \simeq 2 \cdot 10^{-3}$. A higher relaxation rate can be achieved by reducing the time step. However, in our tests, this time step is still several orders of magnitude below the characteristic relaxation time $1 / \Omega$. The validity range of the hypotheses given in Sections 2.3.2 and 3.3 should be investigated further, as well as the stability of the explicit Euler time discretization.

Two variants of the initial condition are used with different interface diffusions to check the robustness of the numerical scheme and the influence of the interface thickness. These initial conditions are shown in Figure 12. The equations of state and initial condition are similar to the test case in Section 4.1 and Table 1 . The magnitudes $\xi$ and $\alpha$ cannot both have symmetrical profiles: it has been chosen to have symmetrical $\alpha$ magnitude to ensure that the total mass of gas and liquid are the same with or without the diffused interface. 

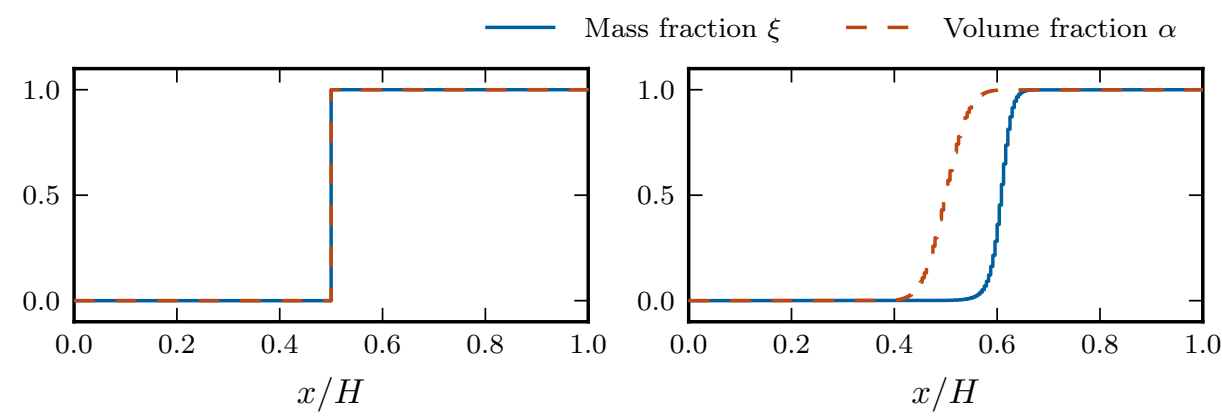

Figure 12: Initial profiles of mass fraction $\xi$ (solid blue lines) and volume fraction $\alpha$ (dashed red lines), for the sharp initial condition (left) and the diffuse initial condition (right). On the left, the mass fraction and the volume fraction curves are superimposed.

Four different initial pressures will be tested for both evaporation $\left(p<p_{0}\right)$ and condensation $\left(p>p_{0}\right)$.

Assuming the pressure to be homogeneous in the gas phase and the volume available for the gas phase to be constant (due to the low density ratio), the dimensionless pressure can be shown to respect:

$$
\frac{\mathrm{d} \tilde{p}}{\mathrm{~d} \tilde{t}}=\Omega(1-\tilde{p}) .
$$

This analytical solution will be compared to the numerical results.

\subsubsection{Results and Discussion}

Figure 13 presents the evolution of the mass and pressure. In all cases, the system relaxes to the $p=p_{0}$ equilibrium state. Both mesh refinement and initial total gas mass. However, the pressure at the center of the domain is slightly affected, although the mean pressure over the whole domain is the same at all resolutions.

The staircase like behavior of the pressure evolution for the cases with a sharp initial interface is caused by pressure waves emitted by the phase change at the interface. These waves take a finite amount of time to travel through the gas phase to the boundary of the domain and return to the interface. 


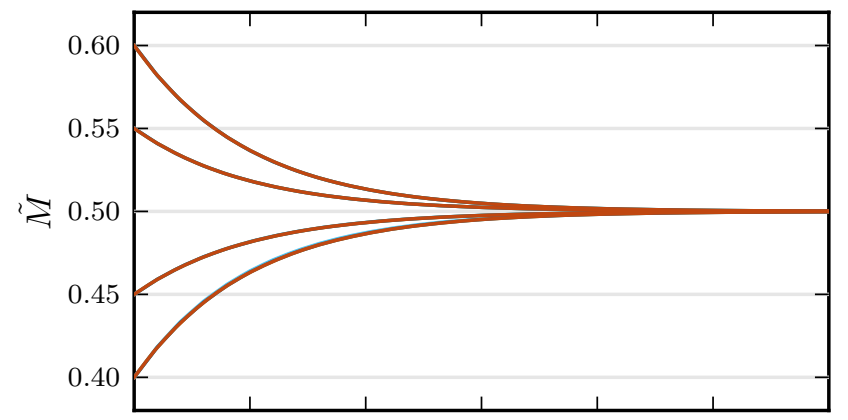
- 120 cells, sharp I.C. 120 cells, diff. I.C. 240 cells, sharp I.C.

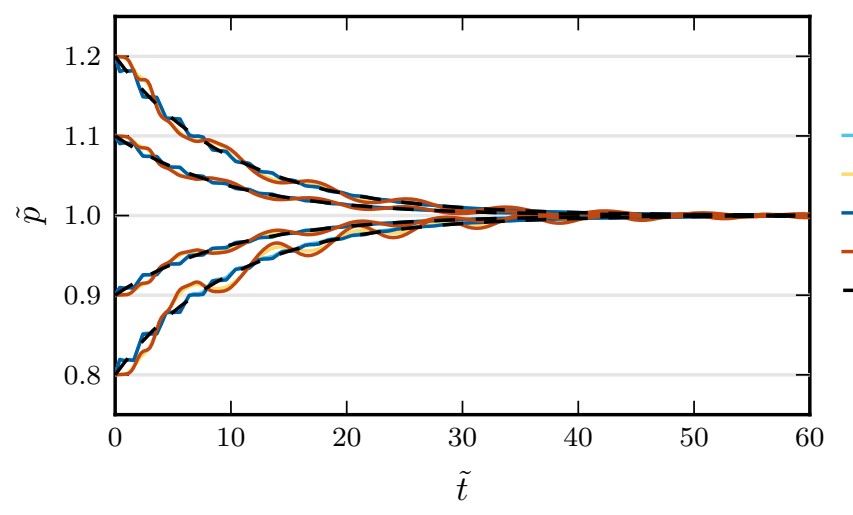

120 cells, sharp I.C. 120 cells, diff. I.C. 240 cells, sharp I.C. 240 cells, diff. I.C. - Analytical solution

Figure 13: Evolution of dimensionless total gas mass (top) and dimensionless pressure at the center of the domain (bottom) as a function of dimensionless time for four different initial pressures, two initial interface diffusions and two different mesh refinements (16 cases in total). The pressures are compared to the analytical solution in (39). The mass evolution curves are superimposed for a given initial pressure.

For a diffuse initial interface the compression wave emitted by the interface is smoother than the sharp shock emitted by the sharp interface. Moreover, the interaction of a pressure wave with a diffuse interface can lead to pressure oscillations inside the diffuse interface, probably due to spatial gradients in the speed of sound. This effect does not seem to influence the phase change relaxation.

The staircase effect would probably not appear experimentally since real ${ }_{645}$ liquid-vapor phase change is not isothermal: it is directed by the thermal diffusion of the latent heat as well as the pressure evolution. 


\section{Application to a simplified 2D impact case}

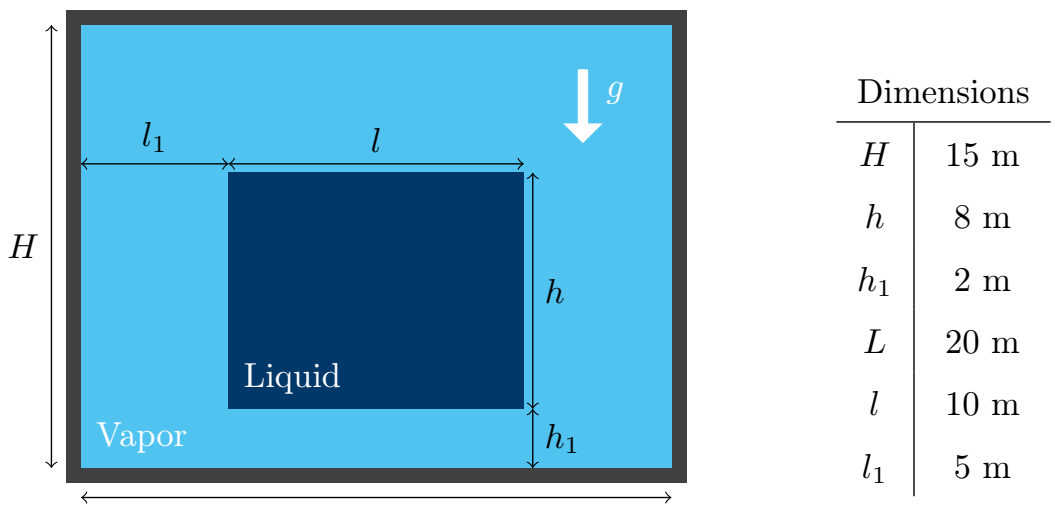

Figure 14: Scheme of the simplified impact test case from [41].

In order to evaluate the effect of phase change on a 2D liquid impact, the numerical test case of [41] is now studied. It is a simple impact scenario consist-

ing of a free falling rectangular liquid block within a much larger rectangular tank of gas (see Figure 14). This test was first proposed by [41] and was used as a benchmark study organized within the ISOPE conferences in 2010 and 2013. Despite its simplicity, this test case shows the different steps of a liquid impact on a solid surface, including the incompressible and then compressible escape of .55 the gas from the space between liquid and wall. Previously, this test case has been studied with phase change by [6].

The fluids are modeled by the same isothermal equations of state (isothermal ideal gas and isothermal stiffened gas) as in the previous test cases. The acceleration due to gravity $\left(g=10 \mathrm{~m} \mathrm{~s}^{-1}\right)$ is modeled classically as a momentum source term on the right hand side of (16) and discretized using the Euler forward method.

For a more efficient numerical resolution of the problem, an adaptive mesh refinement method, based on a quad-tree approach has been used. An example result at the time of maximum pressure is shown in Figure 15 along with the mesh. The mesh is refined when either the norm of the gradient of mass fraction, 
the norm of the gradient of volume fraction or the norm of the gradient of pressure are above an arbitrary threshold. Though the finest mesh cells are $2^{-4} \mathrm{~m}=62.5 \mathrm{~mm}$ in size, the result is still far from mesh convergence. Due to the coarseness of the mesh in comparison with the highest resolution results of [4], for example, and due to the formation of a numerical mixture layer at the interface, a strong mitigation of the impact pressure is observed. Anyway, since here the gas is modeled as isothermal, the impact time and pressure would be different to the reference adiabatic simulations. Nonetheless, we can compare our own simulations with and without phase change.

In Figure 16, the evolution of the pressure at the center of the impact wall is shown for three different values of the phase change coefficient $\Omega$ as defined in (38). Despite the promising properties of the numerical scheme, direct simulation of the higher relaxation rate is not possible, as already mentioned for the previous test case. The dashed lines in Figure 16 are the cases where it was necessary to add the following fix to get an answer:

$$
\xi^{n+1 *}=\min \left(1, \max \left(0, \xi^{n+1}\right)\right) .
$$

Although a supplementary numerical bias might have been introduced in our solutions, this gives a clear idea of the expected qualitative behavior for higher phase change rates. Further work, such as an implicit resolution of the stiff phase change term, may be required here.

A magnification of the pressure peak due to phase change is observed. These results are comparable to the tendencies observed by [6] for the same test case. A more violent impact is observed due to gas vanishing by condensation, which is in addition to gas escaping between the free surface and the wall. Put simply, there is a lower resistance to the falling liquid when more gas escapes, which causes the impact velocity to be higher.

The maximal pressure observed is actually not the pressure of the direct impact of the liquid, but is instead the pressure in a thin gas pocket which is holding the liquid away from the surface, as is visible in Figure 15. The formation of this gas pocket is affected by the entrapment of gas in the diffuse 

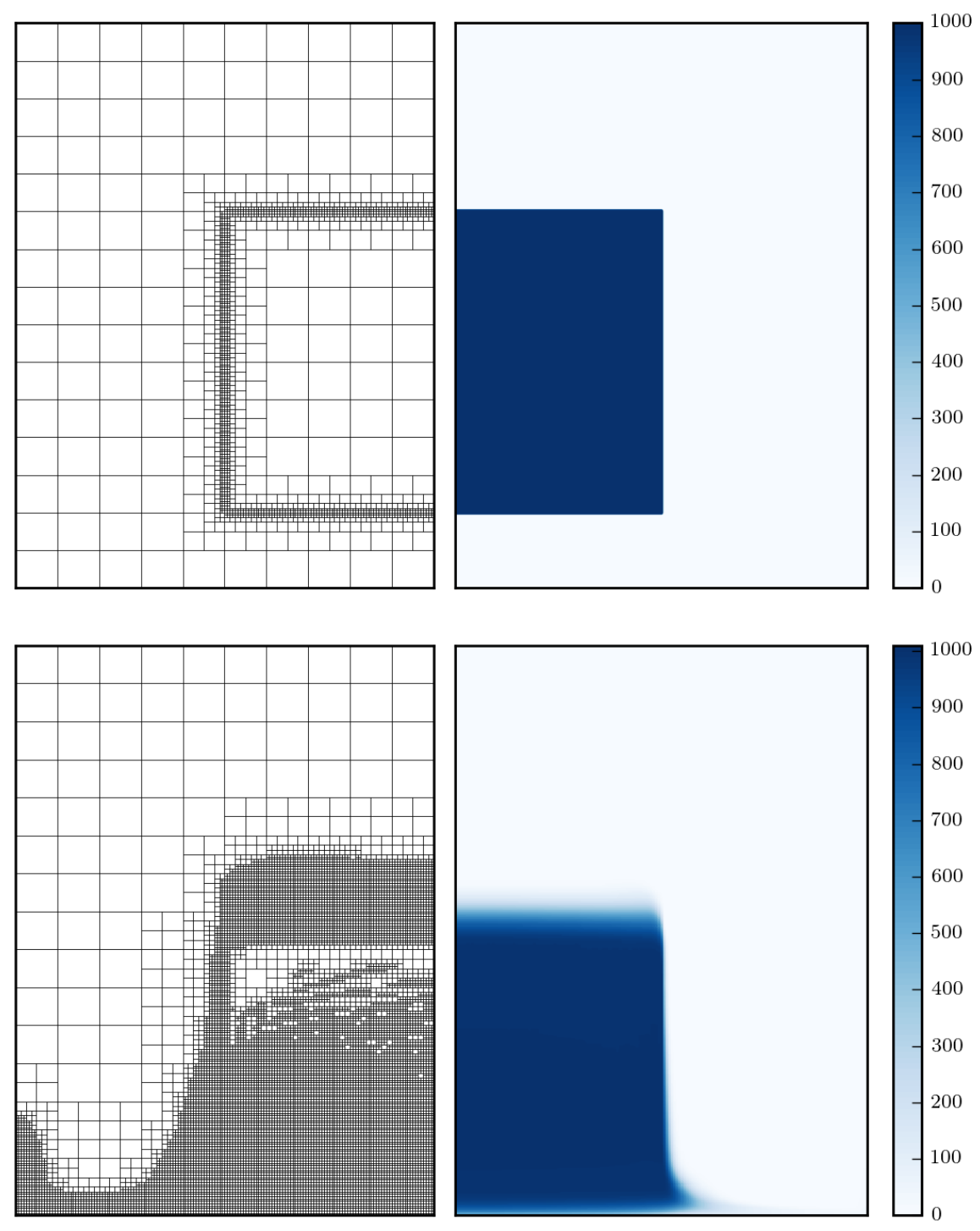

Figure 15: Mesh (left) and density (right) (in $\mathrm{kg} \mathrm{m}^{-3}$ ) initially (above) and at the time of maximum pressure (below). 
interface, but it is not only a numerical artifact as it has been observed in other work, such as [41]. A shortening of the oscillation period of the gas pocket pressure is observed due to phase change. This can be explained by condensation causing a reduction in the volume of trapped gas during the impact. Indeed, for the problem of a piston oscillating on a gas pocket [42], the period of oscillation is shown to change with the size of the gas pocket. The effect of phase change on the piston problem has also been discussed in [24] and [32].

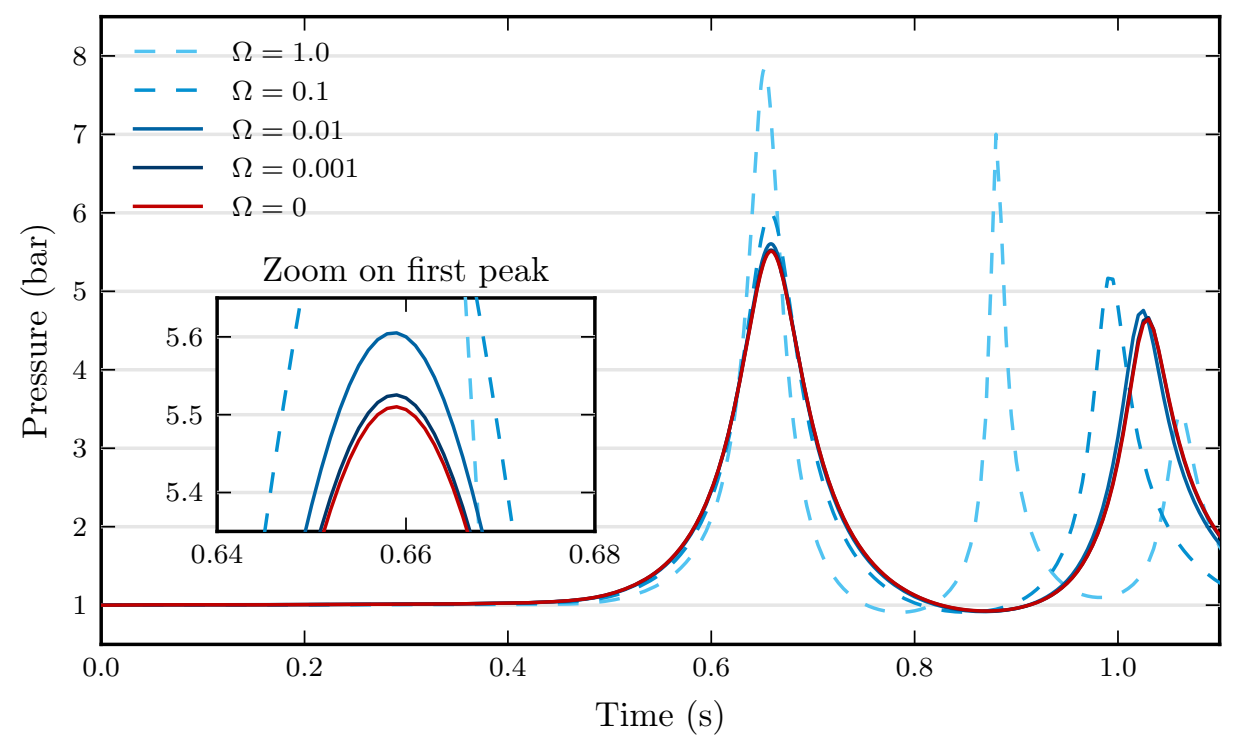

Figure 16: Pressure at the center of the impact wall as a function of time. Without phase change $(\Omega=0)$ and for several relaxation rates $\Omega$ with phase change.

\section{Conclusion}

A physical model that describes a flow with two separate phases including phase change has been presented and an averaged version of the model has then been derived. Unlike other averaged models that are commonly used, phase change is described by a non-conservative advection term. At the interface, this approach limits the influence of the numerical mixture layer thickness on phase 
change.

A Roe-type scheme for the discretization of this model is also presented, tive upwinding matrix, the scheme has improved behavior with respect to the maximum principle of the mass fraction. The alternative matrix allows us to solve stiffer problems than in previous computations of a similar type, such as [20]. Indeed, such models that do not respect the maximum principle discussed tions which cause the simulation to end prematurely, even for constant-velocity advection problems without phase change.

This result has several shortcomings and can only be considered as preliminary. Improvements are still necessary, especially for the stiffest phase change problems, and implicit resolution of the stiff phase change term could be the next step. Also, higher order discretization in space and further study of the automatic mesh refinement criteria could help to improve the quality of the solution with and without phase change.

Thus far, only an isothermal version of the model has been derived and implemented. Such a strong hypothesis is not physically justified for liquidvapor phase change, and particularly not for the problem of liquid impacts on solid walls. This model is nonetheless useful to help understand the behavior of a numerical code when dealing with strong changes in fluid density. The model and discretization presented in this paper are able to simulate relatively densities.

In the final section of this paper, the effect of phase change on a simple wave impact test case has been computed. Phase change causes gas to disappear which results in a higher impact velocity of the liquid on the wall. However, the isothermal hypotheses may cause an overestimation of the magnitude of this effect. According to surrogate models [24], the latent heat may indeed be a limiting factor. The simulations shown in the final section have several other shortcomings (such as relatively high diffusion of the liquid-vapor interface) and 
should therefore be considered as a prototype.

Instead of the isothermal hypothesis, a model with a similar level of complexity could have been built by considering an adiabatic evolution in which the latent heat is artificially set to zero. Such a model would have brought results closer to the usual wave impact models in the case without phase change. However, such a model would be as approximate as the current model when it comes to evaluating the influence of phase change. Besides, the isothermal phase change hypothesis is more common in the literature and it has allowed us to compare our model to [26] in [24].

A further challenge for phase change simulations is dealing with a high latent heat. The mixture model defined in this paper makes the evaluation of the entropy creation at the interface difficult. Thus, it is not easy to distribute the latent heat in the diffused interface in a way that is compatible with the second law of thermodynamics. In [25], a naive extension of (16) that includes the energy equation has nonetheless been used. However, this system has not been rigorously derived and the treatment of the latent heat across a diffuse interface might present some difficulties.

The results presented in this work are in contradiction to the experimental results of [2] discussed earlier, in which a pressure reduction was noticed. In this paper, only a single aspect of the influence of phase change on wave impacts has been discussed: that is the effect on the compressed escaping gas in front of the liquid before the impact. Other works using different approaches $[24,32,33]$ have shown a pressure reduction in the entrapped gas pockets after the impact. Further work is necessary to have a complete description of the total effect of phase change during a complete impact.

Let us finally note that a realistic wave impact involves free surface instabilities, which have not been taken into account in this work. Phase change in aerated liquids leads to a reduction in the acoustic pressure for a given impact velocity. If the developed instabilities are roughly modeled as a layer of aerated liquid, it may then be possible to observe both a increase in the impact velocity, as in Section 5, and a decrease in the maximal pressure. Improved understand- 
ing of the physics of wave impacts in the presence of free surface instabilities is thus needed to make conclusions on the effect of phase change.

\section{Acknowledgment}

This work was carried out during the Ph.D. degree studies of the first author, with the support of GTT and the Association Nationale de la Recherche et de la

775 Technologie (ANRT) through CIFRE contract 2013/1301. The authors thank John Redford (CMLA) for proof-reading the article.

\section{References}

[1] E. Gervaise, P.-E. de Sèze, S. Maillard, Reliability-based methodology for sloshing assessment of membrane LNG vessels, in: Proc. of the 19th Int. Offshore and Polar Eng. Conf., Vol. 3, ISOPE, 2009, pp. 183-191.

[2] S. Maillard, L. Brosset, Influence of density ratio between liquid and gas on sloshing model test results, Int. J. of Offshore and Polar Eng. 19 (4) (2009) 271-279.

[3] W. Lafeber, L. Brosset, H. Bogaert, Elementary loading processes (ELP) involved in breaking wave impacts: findings from the sloshel project, in: Proc. of the 22nd Int. Offshore and Polar Eng. Conf., Vol. 3, ISOPE, 2012, pp. 265-276.

[4] P.-M. Guilcher, Y. Jus, N. Couty, L. Brosset, Y.-M. Scolan, D. Le Touzé, 2d simulations of breaking wave impacts on a flat rigid wall - part 1: influence of the wave shape, in: Proc. of the 24th Int. Offshore and Polar Eng. Conf., Vol. 3, ISOPE, 2014, pp. 232-245.

[5] F. Dias, J.-M. Ghidaglia, Slamming: Recent progress in the evaluation of impact pressures, Annual Review of Fluid Mechanics 50 (2018) 243-273. 
[6] P. Behruzi, M. Konopka, F. de Rose, G. Schwartz, Cryogenic slosh modeling in LNG ship tanks and spacecrafts, in: Proc. of the 24th Int. Offshore and Polar Eng. Conf., Vol. 3, ISOPE, 2014, pp. 209-217.

[7] P. Behruzi, D. Gaulke, D. Haake, L. Brosset, Modeling of impact waves in LNG ship tanks, Int. J. Offshore and Polar Eng. 27 (1) (2017) 18-26.

[8] T. Arndt, M. Dreyer, P. Behruzi, M. Winter, A. vanForeest, Cryogenic sloshing tests in a pressurized cylindrical reservoir, in: 45th AIAA/ASME/SAE/ASEE Joint Propulsion Conference \& Exhibit, 2009, p. 4860 .

[9] D. Jamet, O. Lebaigue, N. Coutris, J. M. Delhaye, The second gradient method for the direct numerical simulation of liquid-vapor flows with phase change, J. Comput. Phys. 169 (2) (2001) 624-651.

[10] D. Bestion, The physical closure laws in the CATHARE code, Nucl. Eng. and Des. 124 (3) (1990) 229-245.

[11] M. Ishii, T. Hibiki, Thermo-fluid dynamics of two-phase flow, Springer New York, 2011.

[12] I. Toumi, A weak formulation of Roe's approximate riemann solver, J. Comput. Phys. 102 (2) (1992) 360-373.

[13] J. G. Collier, J. R. Thome, Convective boiling and condensation, Clarendon Press, 1994.

[14] A. Faghri, Y. Zhang, Transport phenomena in multiphase systems, Elsevier, 2006.

[15] D. Juric, G. Tryggvason, Computations of boiling flows, Int. J. Multiph. Flow 24 (3) (1998) 387-410.

[16] C. Kunkelmann, Numerical modeling and investigation of boiling phenomena, Ph.D. thesis, TU Darmstadt (2011). 
[25] M. Ancellin, L. Brosset, J.-M. Ghidaglia, Preliminary numerical results on the influence of phase change on wave impacts loads, in: Proc. of the 26th Int. Offshore and Polar Eng. Conf., Vol. 3, ISOPE, 2016, pp. 886-893.

[26] M. Hantke, W. Dreyer, G. Warnecke, Exact solutions to the Riemann prob845

[17] J. Stefan, Über die Theorie der Eisbildung, Monatshefte für Mathematik und Physik 1 (1) (1890) 1-6.

[18] R. Marek, J. Straub, Analysis of the evaporation coefficient and the condensation coefficient of water, International Journal of Heat and Mass Transfer 44 (1) (2001) 39-53.

retical investigations on interfacial temperature jumps during evaporation, Experimental Thermal and Fluid Science 32 (1) (2007) 276-292.

[20] F. Dias, D. Dutykh, J.-M. Ghidaglia, A two-fluid model for violent aerated flows, Computers \& Fluids 39 (2) (2010) 283-293.

[21] J. J. Kreeft, B. Koren, A new formulation of Kapila's five-equation model for compressible two-fluid flow, and its numerical treatment, J. Comput. Phys. 229 (18) (2010) 6220-6242.

[22] A. Bernard-Champmartin, F. De Vuyst, A low diffusive Lagrange-remap scheme for the simulation of violent air-water free-surface flows., J. Comput. Phys. 274 (2014) 19-49.

[23] P. D. Hicks, LNG-solid impacts with gas cushioning and phase change, Journal of Fluids and Structures 80 (2018) $22-36$.

[24] M. Ancellin, Sur la modélisation physique et numérique du changement de phase interfacial lors d'impacts de vagues, Ph.D. thesis, École normale supérieure Paris-Saclay (2017).

lem for compressible isothermal Euler equations for two-phase flows with and without phase transition, Q. of Appl. Math. 71 (3) (2013) 509-540. 
[27] C. Rohde, C. Zeiler, A relaxation Riemann solver for compressible twophase flow with phase transition and surface tension, Appl. Numer. Math. 95 (2014) 267-279.

[34] J. M. Delhaye, Jump conditions and entropy sources in two-phase systems. local instant formulation, Int. J. Multiph. Flow 1 (3) (1974) 395-409. 
[35] J.-M. Ghidaglia, F. Pascal, On boundary conditions for multidimensional hyperbolic systems of conservation laws in the finite volume framework, Tech. rep., École normale supérieure de Cachan (2002).

[36] S. Faure, J.-M. Ghidaglia, Violent flows in aqueous foams i: Physical and numerical models, Eur. J. Mech. B/Fluids 30 (4) (2011) 341-359.

[37] G. Witterstein, Sharp interface limit of phase change flows, Adv. in Math. Sci. and Appl. 20 (2) (2010) 585-629.

[38] H. W. Alt, G. Witterstein, Distributional equation in the limit of phase transition for fluids, Interfaces and Free Boundaries 13 (4) (2011) 531-554.

[39] B. Larrouturou, How to preserve the mass fractions positivity when computing compressible multi-component flows, J. Comput. Phys. 95 (1) (1991) 59-84.

[40] L. Zhang, Modélisation, analyse et simulations d'écoulements en thermohydraulique par modèles à six équations, Ph.D. thesis, École normale supérieure Paris-Saclay (2017).

[41] J.-P. Braeunig, L. Brosset, F. Dias, J.-M. Ghidaglia, Phenomenological study of liquid impacts through $2 \mathrm{~d}$ compressible two-fluid numerical simulations, in: Proc. of the 19th Int. Offshore and Polar Eng. Conf., Vol. 3, ISOPE, 2009, pp. 21-29.

[42] L. Brosset, J.-M. Ghidaglia, P.-M. Guilcher, L. Le Tarnec, Generalized Bagnold model, in: Proc. of the 23rd Int. Offshore and Polar Eng. Conf., Vol. 3, ISOPE, 2013, pp. 209-223. 